\title{
Gastos en salud, crecimiento económico y mortalidad infantil: antecedentes de países desarrollados y en desarrollo
}

\author{
Abdelhafidh Dhrifi
}

\section{Resumen}

Este artículo investiga los efectos del gasto en salud sobre las tasas de mortalidad infantil mediante un modelo de ecuaciones simultáneas aplicado a 93 países desarrollados y en desarrollo, utilizando datos que abarcan el período de 1995 a 2012. Las conclusiones a que se llegó muestran que los gastos en salud tienen un efecto positivo de reducción de la mortalidad infantil solo en los países de ingresos medioaltos y altos, mientras que en los de ingresos bajos y medio-bajos el gasto sanitario no tiene un impacto significativo sobre el estado de salud de los niños. También se constató que en los países con un nivel de desarrollo más bajo el gasto público en salud tiene un efecto mayor sobre las tasas de mortalidad que el gasto privado, mientras que cuando el nivel de desarrollo es alto el gasto privado tiene un impacto positivo en la mortalidad infantil.

\footnotetext{
Palabras clave

Salud, economía de la salud, mortalidad infantil, datos estadísticos, modelos econométricos, países desarrollados, países en desarrollo

Clasificación JEL

$\mathrm{H} 51, \mathrm{O} 47, \mathrm{I} 12$

Autor

Abdelhafidh Dhrifi es Doctor en Economía de la Facultad de Economía y Gestión de la Universidad de Susa (Túnez). Correo electrónico: abdelhafidh.dhrifi@gmail.com.
} 


\section{Introducción}

La mortalidad infantil es un fenómeno demográfico muy preocupante - sobre todo en los países en desarrollo - que ha atraído la atención de numerosas partes interesadas, incluidos quienes se encargan de formular las políticas e investigadores. Combatir este flagelo se considera actualmente un objetivo y una estrategia clave en términos de políticas. Al mismo tiempo, algunas organizaciones internacionales como el Fondo de las Naciones Unidas para la Infancia (UNICEF), el Banco Mundial y la Organización Mundial de la Salud (OMS) han incorporado a la mayoría de sus nuevos programas el objetivo de reducir la mortalidad infantil.

De acuerdo con el UNICEF'1 , cada día mueren alrededor de 29.000 niños menores de cinco años, es decir, un promedio de 21 por minuto, principalmente por causas evitables. La mayoría de estas muertes ocurren en los países en desarrollo. Por ejemplo, un niño etíope tiene 30 veces más probabilidades de morir antes de cumplir cinco años que un niño de Europa Occidental. Algunas muertes son consecuencia de enfermedades como sarampión, malaria o tétano, mientras que la desnutrición y la falta de agua potable y saneamiento son un factor determinante en la mitad de todas las muertes infantiles. La investigación y la experiencia muestran que de los casi 11 millones de niños que mueren todos los años, 6 millones podrían salvarse con medidas simples, como vacunas, antibióticos, mosquiteros tratados con insecticida, suplementos de micronutrientes, mejor atención a la familia y lactancia materna. Por lo anterior, reducir la carga de mortalidad y morbilidad entre los pobres se considera hoy uno de los principales desafíos de salud pública a nivel mundial y ha pasado a ser un motivo de gran preocupación de la comunidad internacional, como se reflejó en los Objetivos de Desarrollo del Milenio (ODM) y ahora en los Objetivos de Desarrollo Sostenible de la Agenda 2030 para el Desarrollo Sostenible ${ }^{3}$.

El papel actual de la economía de la salud es decisivo debido a la conciencia internacional cada vez mayor sobre la estrecha relación entre el desarrollo económico y la salud. Además, dado que la salud en la infancia es uno de los principales predictores de la salud y la productividad a lo largo de la vida, la mortalidad infantil es un indicador relevante del desarrollo socioeconómico. La investigación de los factores que subyacen a la elevada mortalidad infantil en el mundo ha revelado diversos elementos que la afectan, como el entorno residencial, la región económica y el nivel de educación de los padres. Por su parte, los países han dado prioridad a las políticas de gasto en salud en vista de que los países con altos niveles de gasto sanitario son los que han conseguido mejores resultados a la hora de reducir las tasas de mortalidad.

Lo anterior sirve de trasfondo a este estudio que, a diferencia del enfoque tradicional, que solo supone el examen de los efectos directos de los gastos en salud sobre la mortalidad infantil, tiene por objeto analizar tanto el impacto directo del gasto sanitario - en términos de hacer posible la satisfacción de las necesidades médicas - como el impacto indirecto, que se hace patente a través del crecimiento económico. El presente artículo también pretende desglosar el gasto sanitario en sus componentes privado y público, a fin de estudiar sus efectos sobre la tasa de mortalidad infantil.

Estudiar esta interacción es importante porque permitirá entender mejor la influencia del gasto sanitario sobre la salud en la infancia. El análisis de la relación entre el gasto en salud, el crecimiento

\footnotetext{
1 Véase Fondo de las Naciones Unidas para la Infancia (UNICEF), "Objetivo: reducir la mortalidad infantil" [en línea] https://www. unicef.org/spanish/mdg/childmortality.html.

2 El Objetivo de Desarrollo del Milenio 4 se refería a la reducción de la tasa de mortalidad de los niños menores de cinco años en dos tercios, desde 93 muertes por cada 1.000 nacidos vivos en 1990 hasta 31 en 2015.

3 Meta 3.2 de los Objetivos de Desarrollo Sostenible: De aquí a 2030, poner fin a las muertes evitables de recién nacidos y de niños menores de 5 años, logrando que todos los países intenten reducir la mortalidad neonatal al menos a 12 por cada 1.000 nacidos vivos y la mortalidad de los niños menores de 5 años al menos a 25 por cada 1.000 nacidos vivos.
} 
económico y la mortalidad infantil hace posible abordar la cuestión del estado de la salud de manera cabal. Así, el verdadero reto al diseñar una política de reducción de la mortalidad infantil es entender estas interacciones.

En este artículo se analiza la relación tripartita en las economías desarrolladas y en desarrollo, comparando los países de ingresos bajos, medio-bajos, medio-altos y altos. Se utilizan datos de panel anuales agregados (de 1995 a 2013), obtenidos de la OMS y de los Indicadores del Desarrollo Mundial sobre gasto en salud y tasas de mortalidad infantil, para una muestra de 93 países desarrollados y en desarrollo, con el objeto de estimar un modelo de las interrelaciones entre el sistema de salud y la tasa de mortalidad de los niños menores de cinco años. Tras esta introducción, el presente documento está organizado como sigue: en la sección II se brinda una reseña de la relación entre los gastos sanitarios y la mortalidad infantil; en la sección III se presentan hechos estilizados sobre las causas de este fenómeno; la sección IV contiene una especificación del modelo, así como una descripción y validación de los datos; en la sección $V$ se exponen un análisis de robustez y un examen de los resultados y, por último, la sección VI incluye las conclusiones y las consecuencias en materia de políticas.

\section{Revisión de la literatura}

En esta sección se examinan los estudios empíricos relacionados del campo de la economía de la salud y se presentan sus principales resultados sobre las interacciones entre el gasto en salud, el crecimiento económico y la mortalidad infantil. Algunos de estos estudios han mostrado que el gasto no tiene ningún impacto en la producción de servicios de atención a la salud, otros ponen de manifiesto un efecto positivo y un tercer grupo revela que el impacto es ambiguo.

\section{A. Efecto nulo del gasto en salud sobre la mortalidad infantil}

Musgrove (1996) y Filmer y Pritchett (1997) presentan argumentos que muestran que el gasto público en salud no tiene influencia en la reducción de la mortalidad infantil. Estos autores consideran que variables como el ingreso, la desigualdad de ingresos, la educación de las mujeres y el grado de fragmentación etnolingüística explican casi toda la variación de las tasas de mortalidad infantil. Sobre la base de estos resultados, sostienen que, antes que aumentar el gasto público en salud, para reducir la mortalidad infantil se deben apoyar políticas que estimulen el crecimiento económico, reduzcan la pobreza y la desigualdad de ingresos y aumenten el nivel de educación de las mujeres. Wagstaff, Van Doorslaer y Watanabe (2003) muestran que la formulación de una política adecuada y la calidad de las instituciones determinan en forma significativa el impacto del gasto público en salud sobre el sector de la producción. Roberts y Bogg (2004) exponen que los niños nacidos en hogares de bajos ingresos tienen una mayor probabilidad de experimentar problemas de desarrollo y de salud desde que nacen y de acumular riesgos sanitarios durante el crecimiento. A pesar de que muchos investigadores han resaltado los vínculos entre pobreza y mortalidad, es necesario reconocer que esta relación opera en ambas direcciones. En ese sentido, Wagstaff (2002) señala que la pobreza produce mala salud y que la mala salud mantiene pobres a los pobres. Además, se deben tener en cuenta las diferencias socioeconómicas en relación con el acceso a los servicios de salud. Existe evidencia de que, teniendo en cuenta sus necesidades, es menos probable que las personas de los grupos socioeconómicos más bajos utilicen estos servicios en comparación con aquellas de ingresos más altos, y es más probable que retrasen la búsqueda de tratamiento. 


\section{B. Gasto en salud y mortalidad infantil: una interacción tanto positiva como negativa}

Durante la última década, algunos estudios han mostrado una relación positiva entre el gasto en salud y el capital de salud (Baldacci y otros, 2004). Otros indican lo contrario (Filmer y Pritchett, 1999), y algunos revelan que los resultados dependen del tipo de datos y métodos de estimación utilizado (Baldacci, Guin-Siu y de Mello, 2003). Una última categoría pone de manifiesto que la contribución del gasto sanitario al estado de salud de la población, medido a partir de la mortalidad infantil y la mortalidad materna, es incluso menos significativo en términos estadísticos (Filmer, Hammer y Pritchett, 1998; Musgrove, 1996).

Sobre la base de información de 50 países en desarrollo y en transición, Gupta y Baghel (1999) llegaron a la conclusión de que el gasto en salud reducía las tasas de mortalidad infantil. Por su parte, utilizando datos específicos de gasto sanitario de 20 países de la Organización de Cooperación y Desarrollo Económicos (OCDE) incurrido durante el período comprendido entre 1960 y 1992, Berger y Messer (2002) descubrieron que las tasas de mortalidad dependían simultáneamente del gasto en salud y del tipo de seguro médico. Más concretamente, un aumento de la proporción del gasto público asignado al gasto sanitario se ha asociado con una reducción de las tasas de mortalidad.

Khaleghian y Das Gupta (2005), en un estudio de más de 70 economías en desarrollo y en transición, demuestran que el gasto en salud pública desempeña un papel de mayor relevancia para los pobres en países de bajos ingresos que en los de ingresos altos, además de poner de relieve que los rendimientos del gasto en salud son mayores en los países de ingresos bajos. Harttgen y Misselhorn (2006) señalan que el acceso a la infraestructura de salud reduce significativamente la mortalidad infantil y que los factores socioeconómicos son los que determinan en mayor medida el estado de salud de los niños (Nolte y McKee, 2004).

Baldacci, Guin-Siu y de Mello (2003) y Gupta, Verhoeven y Tiongson (2002) llegaron a la conclusión de que el gasto social cumple un papel significativo en los sectores de la salud y la educación y también muestran que el gasto en educación tiene un mayor efecto sobre los indicadores de desarrollo humano que el gasto en salud. Esta relación positiva entre gasto social e indicadores de desarrollo humano también fue corroborada por un estudio de Ravallion (1997). Los autores mencionados indican que el gasto público en salud tiene un impacto positivo en el estado de salud de los grupos de ingresos bajos. Bokhari, Gay y Gottret (2007) también señalan que, si bien el crecimiento económico es uno de los principales factores que determinan los resultados en materia de salud en los países en desarrollo, el gasto del gobierno en esta área también es importante. Por último, utilizando datos de las Encuestas de Demografía y Salud, Wang y Wang (2002) examinan los elementos clave para los resultados en materia de salud en países de bajos ingresos, tanto a nivel nacional como en zonas rurales y urbanas, y destacan que el gasto público en salud a nivel nacional puede reducir notoriamente la mortalidad infantil.

Nixon y Ulmann (2006) advierten que los aumentos del gasto público en salud se han traducido en una contribución relativamente marginal a la mayor expectativa de vida en 15 países de la Unión Europea y durante un período de análisis que comprende desde 1980 hasta 1995. Asimismo, a partir de datos transversales de 1993 que abarcan 117 países, Zakir y Wunnava (1997) descubren que el gasto en salud de los gobiernos, como porcentaje del producto nacional bruto (PNB), no es un factor determinante de las tasas de mortalidad infantil.

A raíz de las divergencias existentes entre los hallazgos convendría aclarar estas relaciones en los 93 países desarrollados y en desarrollo de la muestra. Los modelos de ecuaciones simultáneas, que no han sido usados en otros estudios sobre este tema, tienen la ventaja de que permiten separar los efectos directos de una infraestructura de salud de mejor calidad de los efectos indirectos del crecimiento económico. 


\section{Hechos estilizados y causas de la mortalidad infantil}

De acuerdo con el Grupo Interinstitucional para la Estimación de la Mortalidad en la Niñez (2015), la tasa de mortalidad de los niños menores de cinco años disminuyó un 53\% entre 1990 y 2015, pasando de 91 muertes por cada 1.000 nacidos vivos a 43. A nivel mundial, la proporción en que se redujo anualmente esa tasa, en promedio, pasó de un 1,8\% de 1990 a 2000 a un 3,9\% de 2000 a 2015, aunque esto fue insuficiente para lograr el Objetivo de Desarrollo del Milenio 4, que exigía una disminución anual del 4,3\% durante todo el período. Sin embargo, en 2015 murieron alrededor de 19.000 niños menos al día que en 1990 y, entre las regiones en desarrollo, Norte de África, Asia Oriental y América Latina y el Caribe alcanzaron la meta de los ODM en cuanto a reducir la tasa de mortalidad de los niños menores de cinco años en dos tercios o más (véanse los gráficos 1 y 2) ${ }^{4}$. No obstante, incluso en esas regiones, en muchos países no se logró la meta, aun cuando mejoró el ritmo en que se redujo la tasa de mortalidad mencionada (véase el cuadro 1).

\section{Gráfico 1}

Tasa de mortalidad infantil y gasto en salud a nivel mundial, 1995-2012

(En número de niños por cada 1.000 nacidos vivos y billones de dólares)

A. Gasto en salud

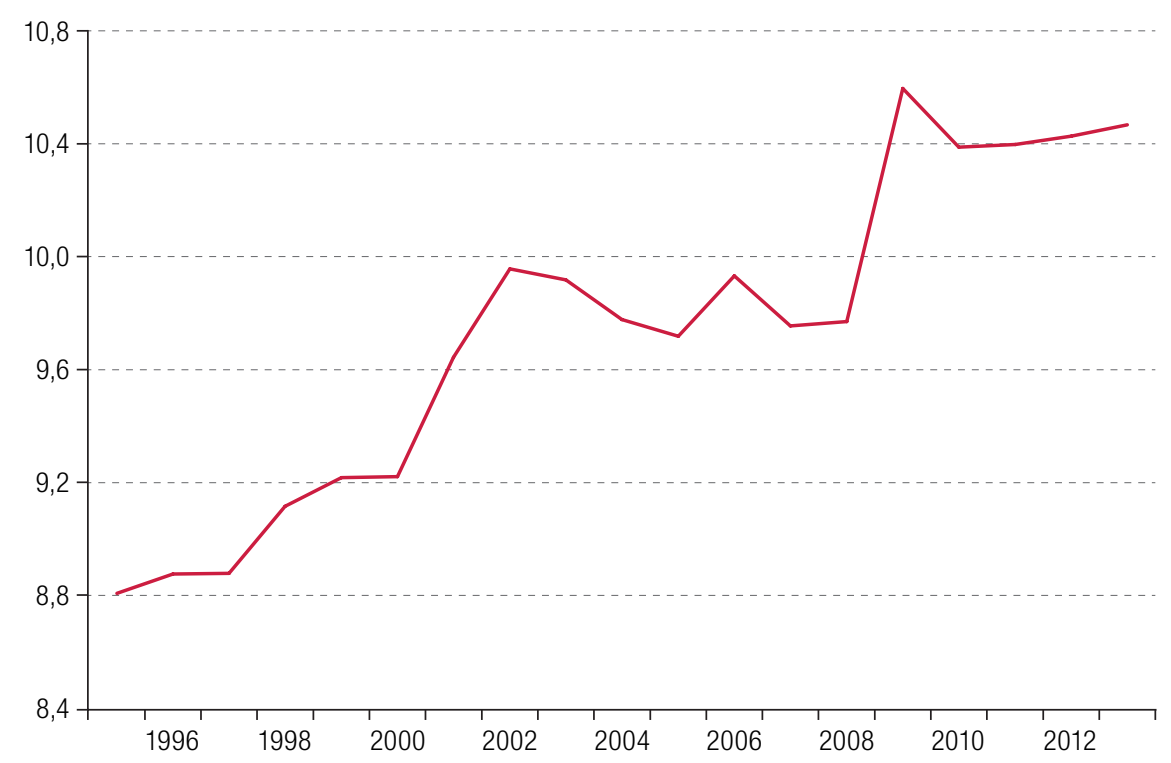

\footnotetext{
4 Los gráficos de este artículo fueron elaborados por el autor a partir de datos del Banco Mundial y de los Indicadores del Desarrollo Mundial.
} 


\section{B. Mortalidad infantil}

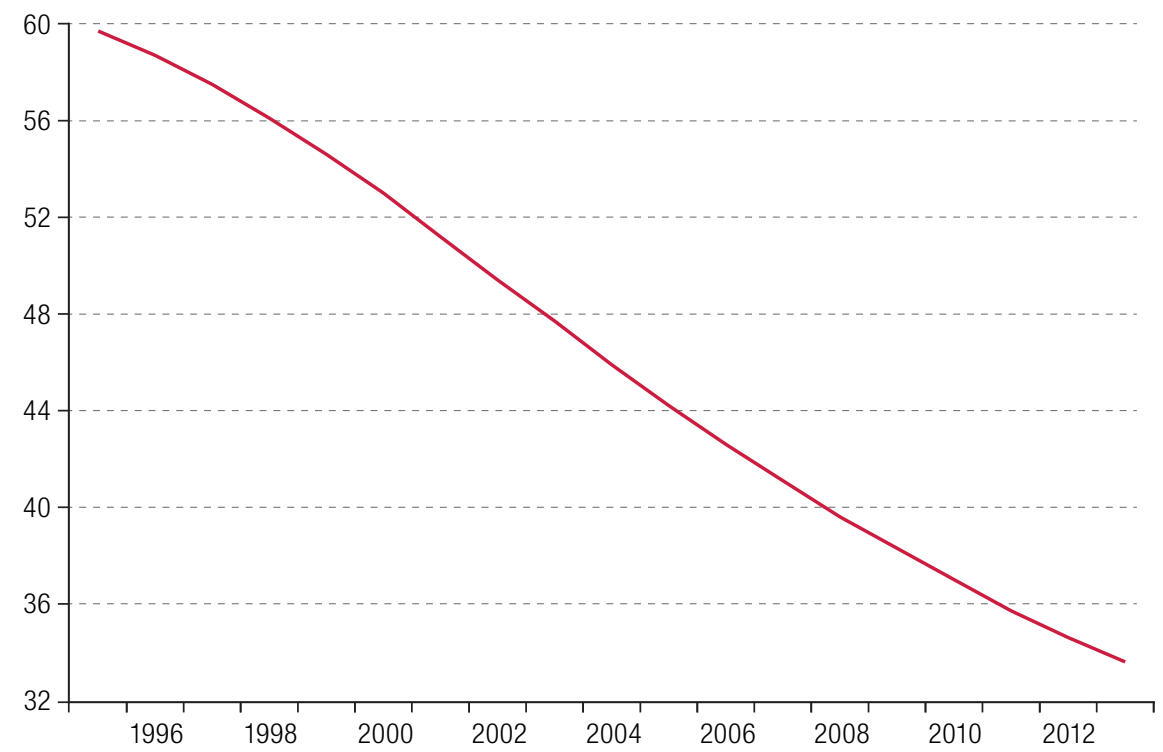

Fuente: Elaboración propia.

Gráfico 2

Mortalidad infantil por región, 1995-2012

(En número de niños por cada 1.000 nacidos vivos)

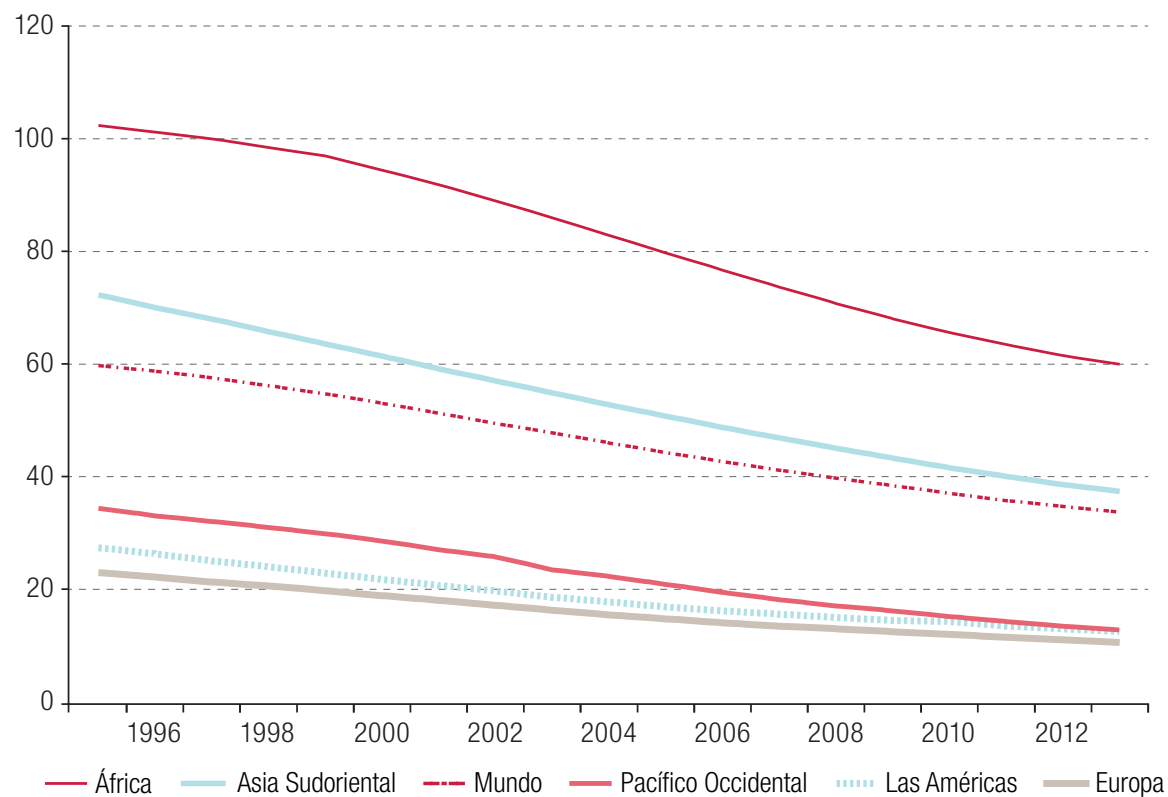

Fuente: Elaboración propia.

La razón entre las diez tasas más altas de mortalidad infantil a nivel nacional y las diez más bajas es de 38. Por ejemplo, en 2015 era 35 veces más probable que muriera un niño afgano que un niño francés antes de cumplir un año. Algunas estimaciones recientes sugieren que cerca del $80 \%$ de las muertes de niños menores de cinco años en el mundo ocurren en África Subsahariana y Asia Meridional, y en 2008 casi la mitad ocurrieron en cinco países: India, Nigeria, República Democrática 
del Congo, Pakistán y China (Black y otros, 2010). En la India y Nigeria en conjunto se produjeron más de un tercio de las muertes de niños menores de cinco años en 2013. El hecho de que una gran proporción de las muertes de niños sea producto de enfermedades infecciosas evitables es sintomático de los sistemas de salud disfuncionales de los países en desarrollo. Por ejemplo, casi la mitad (44\%) de las muertes de niños menores de cinco años a nivel mundial corresponde a recién nacidos. Esto puede deberse a que muchos países de África Subsahariana tienen déficits significativos en términos del acceso a los servicios sociales básicos, como educación y servicios de salud, agua potable, nutrición adecuada, y servicios de higiene y saneamiento. Así, la expectativa de vida al nacer está entre las más bajas del mundo (véanse el cuadro 1 y el gráfico 3), mientras que la tasa de mortalidad infantil disminuyó 21 puntos porcentuales, pasando de 168 muertes por cada 1.000 nacidos vivos en 1990 a 126 en 2013. No obstante, el progreso fue insuficiente para alcanzar la meta establecida conforme al ODM 4 en todo el continente.

\section{Gráfico 3}

Promedios de la mortalidad infantil y la expectativa de vida al nacer en los países de la muestra, 1995-2012

(En número de niños por cada 1.000 nacidos vivos y número de años ${ }^{\text {) }}$

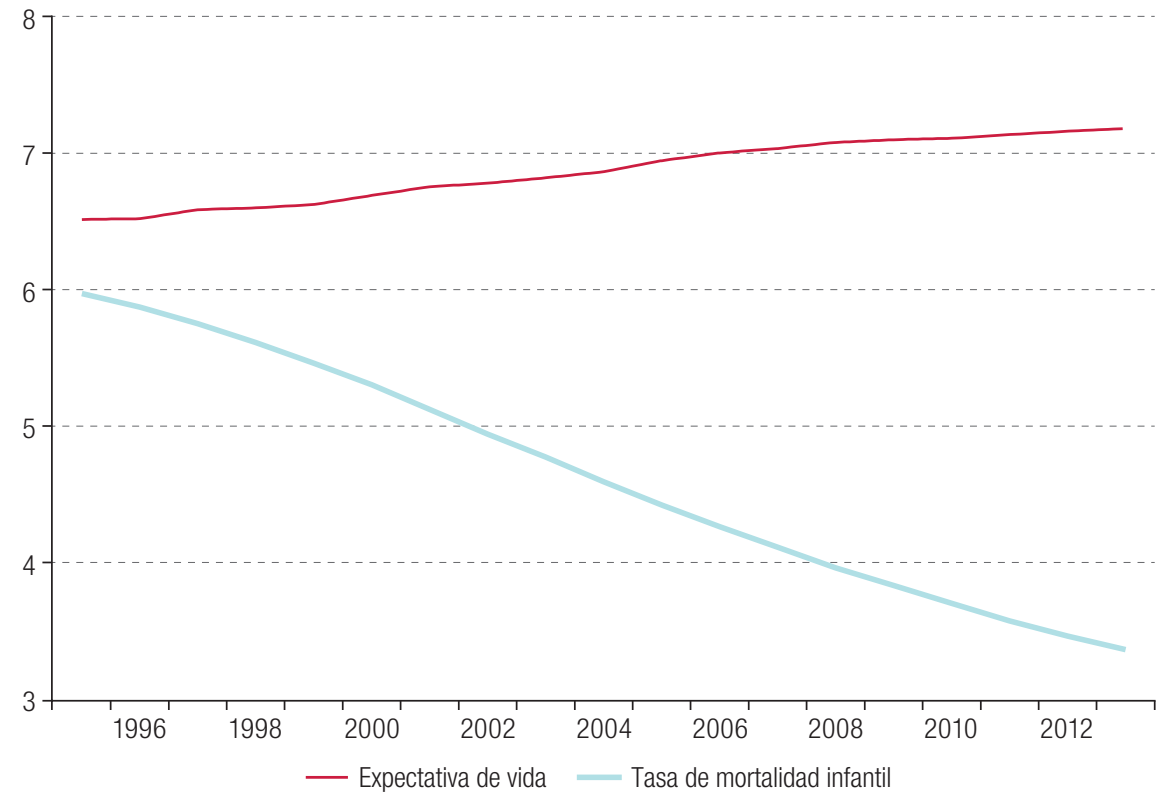

Fuente: Elaboración propia.

a Ambas medidas corresponden a la escala de la izquierda.

Cuadro 1

Expectativa de vida al nacer por región

(En número de años)

\begin{tabular}{lcccc}
\hline & 1990 & 2000 & 2012 & 2013 \\
\hline África & 50 & 50 & 58 & 58 \\
\hline Las Américas & 71 & 74 & 76 & 77 \\
\hline Asia Sudoriental & 59 & 63 & 67 & 68 \\
\hline Europa & 72 & 72 & 76 & 76 \\
\hline Mediterráneo Oriental & 62 & 65 & 68 & 68 \\
\hline Paćfico Occidental & 69 & 72 & 76 & 76 \\
\hline Mundo & 64 & 66 & 70 & 71 \\
\hline
\end{tabular}

Fuente: Organización Mundial de la Salud (OMS), Estadísticas Sanitarias Mundiales, 2015, Luxemburgo, 2015. 
Por otra parte, alrededor del $45 \%$ de todas las muertes de niños menores de cinco años están asociadas a la desnutrición en esas edades, y un niño que se alimenta exclusivamente de leche materna tiene 14 veces menos probabilidades de morir en los primeros seis meses de vida que uno que no recibe ese tipo de alimentación. Los países deben intensificar los esfuerzos destinados a crear un sistema de registro de datos vitales creíble, a fin de mejorar la calidad de la información de salud y aumentar la capacidad de controlar el progreso hacia la meta 3.2 de los Objetivos de Desarrollo Sostenible. En una escala menor, muchos países están atrapados en la trampa de pobreza, que contribuye significativamente al precario estado de la salud. Por consiguiente, se debe considerar la equidad en las políticas de salud pública, que en la actualidad son relativamente desfavorables para los pobres. En los países de ingresos altos, en 2008 el gasto per cápita en salud fue superior a 3.000 dólares en promedio, en comparación con solo 30 dólares per cápita en los países de pocos recursos. De acuerdo con el Banco Mundial, en 2013 más de 50 países gastaron menos de 100 dólares per cápita. Existe también una amplia variación del gasto en relación con el desarrollo económico. Algunos países gastan más del 12\% de su producto interno bruto (PIB) en salud, mientras que otros gastan menos del 3\% (véanse los gráficos 4 y 5).

Gráfico 4

Gasto en salud por región, 1995-2012

(En miles de millones de dólares)

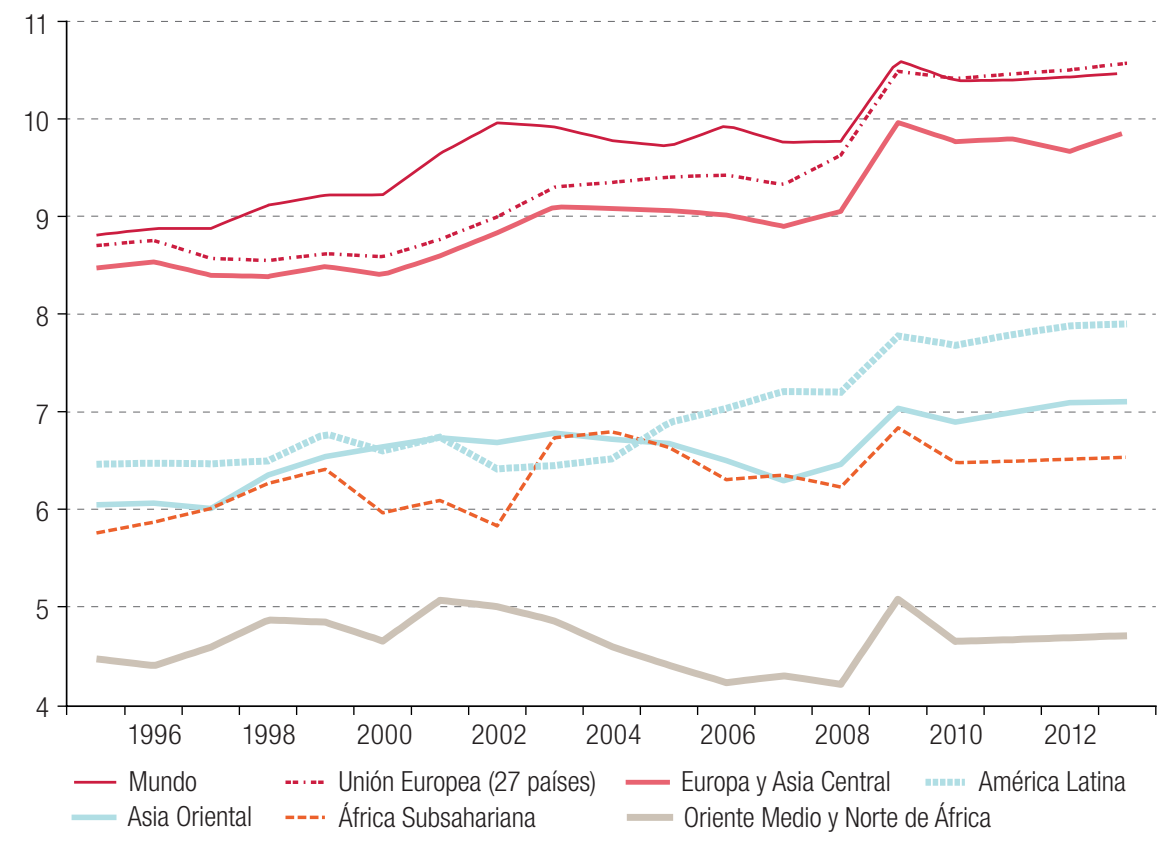

Fuente: Elaboración propia. 
Gráfico 5

Gasto en salud según la clasificación de los países por nivel de ingreso

(En miles de millones de dólares)

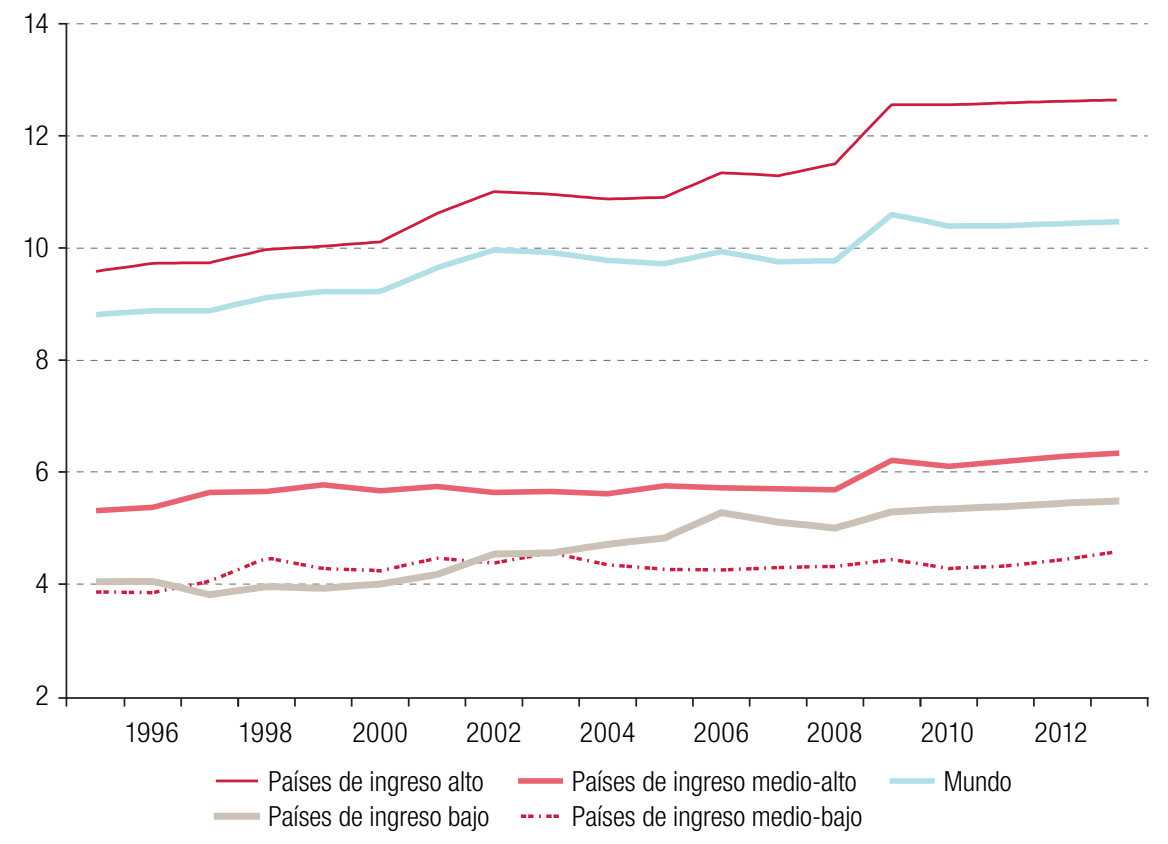

Fuente: Elaboración propia.

\section{Especificación del modelo empírico y descripción de los datos}

\section{A. La modelización econométrica}

El presente artículo tiene por objeto analizar la interrelación entre gasto en salud, crecimiento económico y mortalidad infantil en 93 países desarrollados y en desarrollo, utilizando datos anuales correspondientes al período entre 1995 y 2013. La muestra se eligió sobre la base de dos criterios: primero, la disponibilidad de datos para las variables usadas en el modelo, y, en segundo lugar, la heterogeneidad, con el fin de poder examinar si los efectos del gasto en salud son los mismos en los países desarrollados y en desarrollo.

Las tres variables en cuestión son, en efecto, endógenas. Como se mencionó con anterioridad, en la mayor parte de la literatura generalmente se supone que los gastos en salud promueven el crecimiento económico, pero también se ha establecido que el crecimiento económico es a menudo un factor que determina en gran medida la mortalidad infantil. Por lo tanto, valdría la pena investigar las interrelaciones que existen entre las tres variables, considerándolas en forma simultánea en el marco de una modelización.

Para investigar empíricamente el impacto del gasto en salud sobre la mortalidad infantil, se debe especificar un modelo que permita capturar tanto los efectos directos como los indirectos. En este sentido, se considera que un modelo de ecuaciones simultáneas es el más apropiado porque permite examinar ambos efectos simultáneamente: los directos, relativos a la satisfacción de las necesidades médicas, y los indirectos, que se manifiestan por la vía del crecimiento económico. La especificación 
del modelo es coherente con la literatura y hace posible identificar los canales a través de los que el gasto total en salud y otras variables afectan la salud infantil.

Al analizar los efectos del gasto sanitario en la mortalidad infantil, considerando además el papel del crecimiento económico, deben también dilucidarse las dos variables explicativas (gasto en salud y crecimiento económico). Una razón obvia del carácter endógeno de estas dos variables explicativas en el modelo de regresión es la simultaneidad: en otras palabras, al menos una de las variables explicativas se determina en conjunto con la variable dependiente. Los modelos de este tipo se conocen como modelos de ecuaciones simultáneas.

Hay tres ecuaciones para explicar este fenómeno en su conjunto: una ecuación de mortalidad, una de crecimiento y una de gasto en salud. En este caso no es necesario que todas las variables aparezcan en las tres ecuaciones, de modo que la estimación de los parámetros tiene características que no están presentes en un modelo que solo contenga una relación única. En particular, cuando una relación es parte de un sistema, algunas variables explicativas son estocásticas y están correlacionadas con las perturbaciones. En consecuencia, se viola el supuesto básico de un modelo de regresión lineal, relativo a que la variable explicativa y los términos de perturbación no están correlacionados, o a que las variables explicativas son fijas, y el estimador de mínimos cuadrados ordinarios se vuelve incoherente. Así como las variables de un modelo de regresión lineal se clasifican en explicativas y dependientes, las de un modelo de ecuaciones simultáneas se clasifican en endógenas y exógenas.

Esta distinción es importante porque la condición necesaria para estimar todos los parámetros de forma única es que el número de variables endógenas sea igual al número de ecuaciones independientes del sistema. Además, la característica distintiva de las variables predeterminadas al estimar los parámetros es que no están correlacionadas con los términos de perturbación de las ecuaciones en que aparecen.

En este artículo se presenta la regresión simultánea de las ecuaciones de mortalidad infantil (CHM), crecimiento económico (GDPG) y gasto en salud (HEXP). La primera permite analizar los efectos directos, mientras que las otras dos, los indirectos. Mediante la primera ecuación básica se examina el impacto directo del gasto en salud sobre la tasa de mortalidad infantil, medida a través de la tasa de mortalidad de los niños menores de cinco años. Además de los gastos en salud, la especificación de las ecuaciones de mortalidad infantil incluye otras siete variables que en la literatura sobre economía de la salud se identifican como factores determinantes, a saber, pobreza, crecimiento del PIB per cápita, expectativa de vida, eficiencia en el uso del agua, tasa de alfabetismo de las mujeres, urbanización y emisiones de $\mathrm{CO}_{2}$, que reflejan la limpieza del medio ambiente.

De acuerdo con Barro (1996) y Ravallion (1997), como factores determinantes del crecimiento económico - además de la variable de gasto en salud - se modelizaron un conjunto de variables macroeconómicas ampliamente utilizadas como elementos que explican el crecimiento. Son las siguientes: el coeficiente de Gini (para explorar el efecto de la desigualdad), la inflación, la apertura comercial, el desarrollo financiero, la tasa de inversión y la fuerza de trabajo. En la literatura sobre economía de la salud se admite que la relación entre el crecimiento económico y el estado de la salud es multifacética y bidireccional.

A partir de la literatura existente sobre los componentes que determinan el gasto en salud, el conjunto de variables explicativas incluye el ingreso (PIB) per cápita - que se ha señalado como factor de gran relevancia para explicar las diferencias de nivel y crecimiento de los gastos totales en salud de un país a otro - y la estructura etaria de la población - por ejemplo, la proporción de jóvenes menores de 15 años y de personas mayores (de entre 65 y 75 años) en relación con la población activa o total -, que a menudo se incluye como una covariable en las regresiones de gasto en salud. Estas variables han sido tradicionalmente destacadas como factores que explican en un grado considerable las variaciones del gasto sanitario. Por otra parte, la proporción de médicos, que corresponde al 
número de doctores por cada 1.000 habitantes y se utiliza como medida de la prestación de servicios de salud, puede considerarse un elemento que está detrás de un mayor gasto en salud. Por último, el progreso tecnológico (estimado con el indicador de investigación y desarrollo) y la calidad institucional (medida por el Estado de derecho) también son variables que determinan el gasto sanitario.

El modelo completo utilizado en este estudio para estimar el impacto del gasto en salud sobre la mortalidad infantil se expresa de la siguiente forma:

$$
\begin{aligned}
& C H M_{i t}=\gamma_{0}+\gamma_{1} G D P G_{i t}+\gamma_{2} H E X P_{i t}+\gamma_{3} P O V_{i t}+\gamma_{4} W A T R_{i t}+\gamma_{5} L E X P_{i t} \\
& +\gamma_{6} \mathrm{FemL}_{i t}+\gamma_{7} \mathrm{Urb}_{i t}+\gamma_{8} \mathrm{CO}_{i t}+\xi_{1 i t} \\
& G D P G_{i t}=\alpha_{0}+\alpha_{1} I N Q_{i t}+\alpha_{2} \operatorname{Inf} f_{i t}+\alpha_{3} T R A D E_{i t}+\alpha_{4} F D_{i t}+\alpha_{5} H E X P_{i t} \\
& +\alpha_{6} R I_{i t}+\alpha_{7} L F_{i t}+\xi_{2 i t} \\
& H E X P_{i t}=\beta_{0}+\beta_{1} G D P G_{i t}+\beta_{2} \text { TechProg }_{i t}+\beta_{3} \text { DocDens }_{i t}+\beta_{4} \text { Popstr }_{i t}+\beta_{5} I N S T_{i t}+\xi_{3 i t}
\end{aligned}
$$

\section{B. Descripción de los datos}

La especificación del modelo es coherente con la literatura y permite identificar los canales a través de los que el gasto en salud y otras intervenciones en materia de políticas afectan la mortalidad infantil a lo largo del tiempo. Las diferentes variables recién mencionadas se definen como sigue:

- $\quad$ Tasa de mortalidad (Mortality rate-CHM): se estima por intermedio de la mortalidad de los niños menores de cinco años y mide la proporción de muertes (por cada 1.000 nacidos vivos). Da cuenta del impacto de la situación económica de las madres, su estilo de vida y características, y de la eficacia de los sistemas de salud respecto de la salud maternal y del recién nacido.

- $\quad$ Gastos en salud (Health-care expenditures-HEXP): la variable que se usa en este estudio como referencia del gasto sanitario es el gasto total per cápita, que incluye el gasto del gobierno y el privado. Los países que gastan más en salud seguramente tendrán una mayor expectativa de vida y, por lo tanto, una menor tasa de mortalidad. Conforme a la literatura sobre economía de la salud, un aumento del gasto sanitario supone un acceso más amplio a los servicios de salud y de otro tipo, lo que contribuye a reducir las tasas de mortalidad infantil. Así, mejorar el estado de salud de la población debería promover el crecimiento económico y, en consecuencia, reducir la pobreza; y, dada la relación negativa que existe entre pobreza y mortalidad, esto podría disminuir la tasa de mortalidad infantil ${ }^{5}$.

- $\quad$ Desigualdad (Inequality-INQ): el coeficiente de Gini es el índice sobre desigualdad de ingresos más utilizado y puede referirse al ingreso de los hogares o per cápita. Se espera que tenga una correlación positiva con las variables dependientes, ya que la mayor desigualdad de ingresos de un país refleja un acceso desigual a la salud, la nutrición y otros servicios, que probablemente reducen las tasas de mortalidad.

- Pobreza (Poverty-POV): el indicador que se usa para medir la pobreza es el consumo per cápita (Odhiambo, 2009; Dhrifi, 2014 y 2015), lo que es coherente con la definición propuesta por el Banco Mundial.

\footnotetext{
5 La Comisión sobre Macroeconomía y Salud de la OMS respalda este punto de vista (OMS, 2001).
} 
- $\quad$ Crecimiento del PIB (GDP growth-GDPG): se refiere al crecimiento del PIB per cápita. La ventaja de este indicador es que la información del Banco Mundial abarca a la mayoría de los países durante un extenso período de tiempo.

- $\quad$ Alfabetismo de las mujeres (Female literacy-FemL): representa la tasa de alfabetismo femenino, que se considera un factor clave para el estado de salud de los niños, así como de la población en general (Baldacci, Guin-Siu y De Mello, 2003).

- $\quad$ Acceso al agua (Water access-Water): el acceso al agua potable se mide a través de la proporción de hogares que obtienen agua considerada potable desde una llave o de pozos tradicionales o de perforación que estén protegidos. Los indicadores consideran las condiciones de acceso, en este caso la distancia a la fuente de agua, cuando la información pertinente está disponible.

- Degradación ambiental (Environmental degradation- $\mathrm{CO}_{2}$ ): se calcula utilizando las emisiones de $\mathrm{CO}_{2}$ per cápita expresadas en toneladas métricas, que reflejan la limpieza del medio ambiente.

- $\quad$ Esperanza de vida al nacer (Life expectancy at birth-Life EXP): se refiere al número promedio de años que se espera que viva un recién nacido si los patrones de mortalidad en el momento del nacimiento permanecen constantes en el futuro (Banco Mundial).

- $\quad$ Fuerza de trabajo (Labour force-LF): corresponde al número de personas empleadas más las que se encuentran desempleadas y están buscando trabajo.

- Urbanización (Urbanization-URB): la proporción de la población urbana con respecto a la población total se utiliza como indicador de la urbanización. Roberts (2003) ha puesto énfasis en que los factores geográficos y demográficos, como tipo de emplazamiento (rural o urbano) y porcentaje de población de esos lugares, afectan los resultados en materia de salud (Schultz, 1993; Baldacci y otros, 2004).

- Inflación (Inflation-INF): la inflación se refiere al crecimiento del índice de precios al consumidor, acerca de lo cual existen datos en el Banco Mundial. Este elemento se incluye en el modelo para capturar el impacto de la estabilización macroeconómica en el crecimiento.

- $\quad$ Comercio (Trade-TRA): esta variable representa la apertura comercial y se define como la suma de las exportaciones y las importaciones en relación con el PIB. Se incluye en el modelo con el fin de reflejar el grado de apertura al comercio internacional.

- Desarrollo financiero (Financial development-FD): se mide a través de la razón entre el crédito interno al sector privado y el PIB. Se espera que exista una relación positiva y significativa entre el indicador de desarrollo financiero y el crecimiento económico.

- $\quad$ Progreso tecnológico (Technological progress-TechProg): se refiere al gasto en investigación y desarrollo (como porcentaje del PIB). Scheffler y otros (2009) señalan que los costos de la salud están aumentando debido a la tecnología. En muchos países industrializados, el ingente crecimiento del gasto en salud está relacionado indirectamente con este factor.

- $\quad$ Tasa de inversión (Investment rate-IR): corresponde a la formación bruta de capital (como porcentaje del PIB), que debería tener efectos positivos en el crecimiento económico.

- Estructura etaria de la población (Population age structure-PopStr): este factor se incluye a menudo como una covariable en las regresiones de gasto en salud. Entre los indicadores que se emplean comúnmente se encuentra la proporción de jóvenes (menores de 15 años) respecto de la población activa.

- $\quad$ Proporción de médicos (Physicians density-DocDens): se refiere al número de doctores por cada 1.000 habitantes y se usa para medir la disponibilidad de servicios de salud en un país. Un número mayor de médicos en relación con la población total refleja un acceso más fácil a la atención de salud y debería estar correlacionado con una menor tasa de mortalidad. 
- Instituciones (Institutions-INST): este índice refleja la calidad de las instituciones, medida por el orden público, también llamado Estado de derecho. Se basa en la Guía internacional de riesgo país (International Country Risk Guide), en que se evalúa la solidez e imparcialidad del sistema legal y la observancia de la ley por parte de las personas.

Por último, en este estudio se usan datos de series de tiempo anuales, que abarcan el período de 1995 a 2013, correspondientes a una muestra de 93 países desarrollados y en desarrollo. La información se obtuvo de varias fuentes, como las series compiladas por la OMS y el Banco Mundial, y de la base de datos de estadísticas financieras internacionales del Fondo Monetario Internacional (FMI). El tamaño de la muestra y el período de estudio están limitados por la disponibilidad de datos para las variables de control.

En el cuadro 2 se presenta un resumen de las estadísticas descriptivas de las variables utilizadas en el análisis empírico. Entre las variables de interés, la tasa de mortalidad infantil de los países de la muestra fue, en promedio, de 42,5 por cada 1.000 nacidos vivos y osciló entre un mínimo de 2,1 y un máximo de 192. En el caso del crecimiento del PIB per cápita, estos países exhibieron un promedio de $0,02 \%$ durante el período considerado, siendo de un 9,3\% el valor más alto y de un -2,0\% el más bajo. Con respecto a la tercera variable de interés, el gasto sanitario, los países gastaron en salud entre el $3,8 \%$ y el $12,3 \%$ del PIB, y el promedio fue del $7,8 \%$.

Cuadro 2

Estadísticas descriptivas

\begin{tabular}{lcrrrr}
\hline & $\begin{array}{c}\text { Número de } \\
\text { observaciones }\end{array}$ & Valor mínimo & Valor máximo & Valor promedio & Desviación estándar \\
\hline CHM & 1767 & 2,10 & 192,00 & 42,500 & 6,452 \\
\hline GDPG & 1767 & $-2,04$ & 9,30 & 0,022 & 0,047 \\
\hline HEXP & 1767 & 0,00 & 0,28 & 0,142 & 0,022 \\
\hline Water & 1767 & 0,04 & 0,67 & 0,390 & 0,571 \\
\hline POV & 1767 & 0,13 & 1,38 & 0,676 & 0,147 \\
\hline Life EXP & 1767 & 55,70 & 83,60 & 71,500 & 4,654 \\
\hline FemL & 1767 & 14,16 & 99,88 & 84,530 & 2,314 \\
\hline URB & 1767 & 1,87 & 100,00 & 53,850 & 1,089 \\
\hline CO 2 & 1767 & 0,10 & 46,70 & 5,990 & 3,427 \\
\hline INQ & 1767 & 0,00 & 0,67 & 0,039 & 0,047 \\
\hline INF & 1767 & $-0,91$ & 8,99 & 1,625 & 3,874 \\
\hline TRA & 1767 & 0,00 & 2,21 & 0,735 & 0,334 \\
\hline FD & 1767 & 8,20 & 174,30 & 66,450 & 8,265 \\
\hline IR & 1767 & 0,62 & 2,63 & 1,055 & 0,153 \\
\hline LF & 1767 & 0,08 & 0,34 & 0,016 & 0,026 \\
\hline TechProg & 1767 & 0,00 & 4,04 & 0,374 & 0,456 \\
\hline DocDens & 1767 & 0,03 & 1,00 & 0,590 & 0,293 \\
\hline PopStr & 1767 & 0,00 & 4,04 & 0,374 & 0,456 \\
\hline INST & 1767 & 0,00 & 1,00 & 0,550 & 0,012 \\
\hline Fuente: & & & & \\
\hline
\end{tabular}

Fuente: Elaboración propia. 


\section{Técnicas de estimación}

Los modelos de ecuaciones simultáneas se emplean normalmente cuando la variable endógena de una ecuación pasa a ser una variable exógena de otra. Los métodos de estimación que se pueden utilizar en el contexto de los modelos de ecuaciones simultáneas dependen de los criterios de identificación ${ }^{6}$ para estimarlos y del problema de la endogeneidad. En este caso, el modelo está sobreidentificado, de modo que el uso de mínimos cuadrados ordinarios (MCO) para calcular las ecuaciones estructurales daría lugar a estimaciones incongruentes respecto a los parámetros del modelo. Por ende, este afronta un problema de endogeneidad de segundo orden, por lo que una estimación mediante MCO significaría un doble registro. El presente método de cálculo se basa en la técnica de mínimos cuadrados en tres etapas (MC3E), que tiene por objeto resolver los problemas de endogeneidad introduciendo las variables problemáticas como variables instrumentales. El tratamiento con STATA 13 proporciona una solución mediante el uso del método de MC3E? .

\section{Exposición y análisis de los resultados principales}

En el cuadro 3 se exponen los resultados de las estimaciones obtenidas mediante el modelo de ecuaciones simultáneas utilizando el método de MC3E para el período comprendido entre 1995 y 2013. La primera columna muestra los resultados de la ecuación de mortalidad infantil, en que los parámetros de interés son los coeficientes que representan el efecto del gasto en salud y el crecimiento del PIB en la mortalidad infantil. El coeficiente estimado respecto del gasto en salud, que incluye tanto el gasto público como el privado, exhibe una clara relación positiva entre esa variable y la mortalidad infantil. El valor de $\gamma_{3}$ de este modelo mide la elasticidad de la mortalidad infantil con respecto al gasto en salud. El coeficiente de $-0,17$ indica que un aumento de este gasto de 1 unidad se traduce en una reducción de la mortalidad infantil de 0,17 unidades, lo que sugiere que un incremento del gasto sanitario per cápita supone un acceso más amplio a la atención de salud y a otros servicios que contribuyen a disminuir las tasas de mortalidad de los niños menores de cinco años.

Los resultados demuestran también que el crecimiento del ingreso per cápita tiene un efecto significativo en la reducción de la mortalidad infantil: un aumento del 1\% se traduce en una disminución de esta última de 0,76 puntos porcentuales. Las tasas de mortalidad de los niños menores de cinco años que son bajas parecen sugerir que el crecimiento económico redunda en una mortalidad infantil inferior. Se espera que una tasa de crecimiento alta resuelva problemas relacionados con la inseguridad alimentaria, el mal estado de los edificios y el equipamiento, la falta de una infraestructura social adecuada y el presupuesto insuficiente para reducir la mortalidad infantil y materna. Además, los ingresos altos dan lugar a una mejor infraestructura de la salud pública, como la relativa al agua y el saneamiento, junto con una mejor nutrición, mejores viviendas y la capacidad de pagar por la atención de salud (Pritchett y Summers, 1996; Cutler, Deaton y Lleras-Muney, 2006).

\footnotetext{
6 Para determinar si una ecuación estructural de un sistema de ecuaciones simultáneas lineales está identificada, se puede usar la siguiente regla: $\mathrm{G}$ = número total de variables endógenas del modelo, es decir, de todas las ecuaciones que este incluye, y $\mathrm{K}=$ número total de variables, endógenas y exógenas, excluidas de la ecuación cuya identificación se quiere evaluar. La condición de orden es la siguiente: si $K=G-1$, la ecuación está exactamente identificada; si $K>G-1$, la ecuación está sobreidentificada, y si $\mathrm{K}<\mathrm{G}-1$, la ecuación no está identificada.

7 Véanse los detalles del método utilizado en Bourbonnais (2002).
} 


\section{Cuadro 3}

Efectos del gasto en salud sobre la mortalidad infantil (en toda la muestra)

\begin{tabular}{|c|c|c|c|}
\hline Variables & $\begin{array}{c}\text { Ecuación de } \\
\text { CHM }\end{array}$ & $\begin{array}{l}\text { Ecuación de } \\
\text { GDPG }\end{array}$ & $\begin{array}{c}\text { Ecuación de } \\
\text { HEXP }\end{array}$ \\
\hline \multirow{2}{*}{ GDPG } & $-0,761$ & - & 0,361 \\
\hline & $(-3,7)^{\star \star \star}$ & - & $(4,76)^{\star \star \star}$ \\
\hline \multirow{2}{*}{ HEXP } & $-0,171$ & 0,472 & - \\
\hline & $(-2,69)^{\star \star}$ & $(3,46)^{\star \star \star}$ & - \\
\hline \multirow{2}{*}{ POV } & 1,052 & - & - \\
\hline & $(4,46)^{\star \star}$ & - & - \\
\hline \multirow{2}{*}{ Water } & $-0,381$ & - & - \\
\hline & $(-1,8)^{\star}$ & - & - \\
\hline \multirow{2}{*}{ Life EXP } & $-1,325$ & - & - \\
\hline & $(2,76)^{\star \star \star}$ & - & - \\
\hline \multirow{2}{*}{ FemL } & $-2,651$ & - & - \\
\hline & $(4,18)^{\star \star \star}$ & - & - \\
\hline \multirow{2}{*}{ URB } & $-0,0364$ & - & - \\
\hline & $(-3,3)^{\star \star}$ & - & - \\
\hline \multirow{2}{*}{$\mathrm{CO}_{2}$} & 0,23 & - & - \\
\hline & $(4,32)^{\star *}$ & - & - \\
\hline \multirow{2}{*}{ INQ } & - & 0,724 & - \\
\hline & - & $(1,88)^{\star \star}$ & - \\
\hline \multirow{2}{*}{ INF } & - & $-0,266$ & - \\
\hline & - & $(-1,72)^{\star}$ & - \\
\hline \multirow{2}{*}{ TRA } & - & 0,364 & - \\
\hline & - & $(1,96)^{\star *}$ & - \\
\hline \multirow{2}{*}{ FD } & - & 0,455 & - \\
\hline & - & $(1,46)$ & - \\
\hline \multirow{2}{*}{$\mathbb{I R}$} & - & 1,452 & - \\
\hline & - & $(5,84)^{\star \star \star}$ & - \\
\hline \multirow{2}{*}{ LF } & - & 0,768 & - \\
\hline & - & $(1,87)^{\star \star}$ & - \\
\hline \multirow{2}{*}{ TechProg } & - & - & 2,263 \\
\hline & - & - & $(1,89)^{\star \star}$ \\
\hline \multirow{2}{*}{ DocDens } & - & - & 0,095 \\
\hline & - & - & $(4,49)^{\star \star}$ \\
\hline \multirow{2}{*}{ PopStr } & - & - & 2,521 \\
\hline & - & - & $(2,05)^{\star \star \star}$ \\
\hline \multirow{2}{*}{ INST } & - & - & 3,489 \\
\hline & - & - & $(5,24)^{\star \star \star}$ \\
\hline \multirow{2}{*}{ Constante } & 5,974 & 7,562 & 3,887 \\
\hline & & $(6,75)^{\star \star}$ & $(6,54)^{\star \star \star}$ \\
\hline Número de observaciones & 1767 & 1767 & 1767 \\
\hline $\mathrm{R}^{2}$ & 0,2 & 0,19 & 0,17 \\
\hline
\end{tabular}

Fuente: Elaboración propia.

Nota: * significativo al 10\%; ${ }^{\star *}$ significativo al 5\%; *** significativo al $1 \%$.

Todas las variables explicativas tienen el signo previsto y son estadísticamente significativas; por ejemplo, el coeficiente estimado para la pobreza muestra que una reducción de la tasa de pobreza de 1 punto porcentual permite que disminuya 1,05 puntos la tasa de mortalidad infantil. Este resultado es coherente con la literatura sobre economía de la salud que plantea que la pobreza es un factor clave de la mortalidad en general, sobre todo en los países en desarrollo. En efecto, la mala salud puede ser un catalizador de las espirales de pobreza, y la pobreza, a su vez, puede generar y perpetuar una condición de salud precaria. Los hogares pobres de los países en desarrollo 
son especialmente vulnerables, y los períodos de mala salud pueden considerarse inherentes a la experiencia de la pobreza. Las enfermedades graves empujan a las personas a la pobreza porque las obligan a abandonar el mercado laboral. La salud es un bien económico de crucial importancia para los pobres, ya que de ella depende su sustento. Cuando una persona pobre o socialmente vulnerable se enferma o sufre una lesión, todo el hogar puede quedar atrapado en una espiral descendente de pérdida de ingreso y altos costos de atención de salud. Los pobres son más vulnerables a esta espiral descendente porque también lo son a las enfermedades y tienen un acceso más limitado a la atención de salud y a la seguridad social. Esto quiere decir que el desarrollo de un país depende de que mejore la salud de la población. Así, el tener un sistema de salud bien desarrollado contribuirá a que aumente la productividad laboral, lo que da lugar al crecimiento de los salarios y del PIB y, en consecuencia, en una menor tasa de pobreza.

El coeficiente estimado relativo a la tasa de alfabetismo de las mujeres parece ser significativamente negativo - lo que sugiere que esta variable determina en alto grado la salud del hogar -, siendo su impacto igual al de la atención prenatal y posnatal en los países en desarrollo. Lo anterior se relaciona con las conclusiones a las que llegaron Baldacci, Guin-Siu y de Mello (2003) y Schultz (1993) acerca de que el nivel de educación de la madre es un factor decisivo para la condición de salud tanto de los niños como de la población en general. En los países en desarrollo, las mujeres desempeñan un papel más importante en la salud e higiene de la familia, aparte de que la educación de la mujer se asocia de manera positiva con la salud infantil y en forma negativa con las tasas de fecundidad.

En el caso de la urbanización, el coeficiente muestra que un aumento de la población urbana de 1 punto porcentual se reflejará en una disminución de la tasa de mortalidad infantil de 0,3 puntos porcentuales. En este contexto, Schultz (1993) indica que esta tasa es más alta en los hogares rurales, de bajos ingresos y agrícolas que en aquellos de zonas urbanas, en parte debido a que la atención de salud es normalmente más accesible en estas zonas y a que el costo privado de acceder a la salud (por ejemplo, el costo de transporte) podría también ser menor para los hogares de áreas urbanas.

La variable medioambiental, medida a través de las emisiones de $\mathrm{CO}_{2}$, exhibe un coeficiente de 0,23 , significativo al $5 \%$, es decir, un aumento de 1 punto porcentual de las emisiones de $\mathrm{CO}_{2}$ se asocia con un incremento de la mortalidad infantil de 0,23 puntos porcentuales.

En la segunda columna del cuadro 3 se observan las estimaciones relativas a la ecuación de crecimiento económico, que muestran una relación positiva entre el gasto en salud y el crecimiento económico. El valor de $\alpha_{5}$ de este modelo, que mide la elasticidad del crecimiento económico con respecto al gasto en salud, es estadísticamente significativo al 5\%, lo que indica que la mala salud es un importante obstáculo al crecimiento del PIB. Una mejor salud permite que aumente la productividad laboral al disminuir el número de días perdidos por licencias médicas, incapacidad o discapacidad. Por otra parte, los trabajadores más saludables tienen más fuerza física y mental y, por lo tanto, son más eficaces dentro del mercado laboral. La mejora de la salud y de los índices sanitarios de la sociedad redundará en un mayor ahorro de las personas, dada la reducción de las tasas de mortalidad y la mayor expectativa de vida.

La variable de desigualdad de ingresos, medida por el coeficiente de Gini, tiene el signo esperado y es estadísticamente significativa: un incremento de la desigualdad de ingresos de 1 punto porcentual se traduce en una reducción del crecimiento del PIB de 0,7 puntos porcentuales, lo que afecta negativamente las tasas de mortalidad infantil. En la literatura especializada se afirma que, si el ingreso se redistribuye desde los ricos, cuya salud no se ve afectada en forma considerable, hacia los pobres, cuya salud es más sensible a sus ingresos, la condición de salud de la población experimentará, en promedio, una mejora. En igualdad de circunstancias, incluido el ingreso medio, los países (u otros grupos de población) que tienen una distribución del ingreso más equitativa tendrán, en promedio, un mejor estado de salud. 
Además, los resultados señalan que la inflación tiene un efecto relevante, de tipo negativo, sobre los procesos de reducción de la pobreza, pues se encontró que un alza del 1\% del índice de precios al consumidor produce una disminución del crecimiento económico de 0,26 puntos porcentuales. Estas cifras son coherentes con la teoría planteada por Kpodar (2004), conforme a la cual la inflación se considera un obstáculo al crecimiento debido a su impacto negativo en el valor real de los activos y del poder adquisitivo del ingreso de los hogares.

La variable de apertura comercial aparece con un valor positivo muy alto, lo que sustenta la idea de que una política de eliminación de las barreras comerciales y promoción de la libre circulación de capitales favorece el crecimiento económico.

La tercera columna del cuadro 3 muestra los resultados obtenidos de la ecuación de gasto en salud. Como se esperaba, las estimaciones reflejan que el gasto sanitario presenta una relación positiva e importante en relación con el crecimiento del PIB per cápita. Ello se ajusta a las predicciones teóricas: si el gasto en salud puede ser considerado una inversión en capital humano, que a su vez es fuente crucial de crecimiento económico, un aumento del gasto sanitario debe en último término redundar en un PIB más alto y viceversa.

El coeficiente del gasto en investigación y desarrollo, que representa el progreso tecnológico, indica que es un elemento clave del gasto en salud de los gobiernos.

Respecto del coeficiente sobre estructura de la población, es estadísticamente significativo al $1 \%$, con lo que se demuestra que esta variable tiene una correlación positiva de gran magnitud con el gasto en salud. Esto quiere decir que el acceso a la atención de la salud tiene un impacto positivo notorio sobre el gasto sanitario. La variable que corresponde a la proporción de médicos también es estadísticamente significativa al 5\%; en este sentido, un mayor número de doctores por cada 1.000 habitantes indica que la atención de salud es más accesible y debería estar correlacionado con una menor tasa de mortalidad.

\section{A. Cálculo del efecto total del gasto en salud sobre las tasas de mortalidad infantil}

Como ya se mencionó, el principal objetivo de este artículo es examinar si el gasto en salud tiene un impacto en la reducción de la mortalidad infantil mediante una influencia positiva sobre el crecimiento económico, así como evaluar la importancia de ese efecto. En términos matemáticos, los efectos directos e indirectos de los gastos sanitarios en la tasa de mortalidad de los niños menores de cinco años se pueden expresar calculando la derivada matemática de la mortalidad infantil (CHM) con respecto al gasto en salud (HEXP):

$$
\frac{\partial C H M}{\partial H E X P}=\gamma_{1} \frac{\partial G D P G}{\partial H E X P}+\gamma_{2}=\gamma_{1} * \alpha_{5}+\gamma_{2}
$$

El cuadro 4 contiene un resumen de las conclusiones acerca del impacto de los gastos en salud en la mortalidad infantil. En términos del impacto directo, un aumento del gasto sanitario de 1 punto porcentual conduce a una disminución de la tasa de mortalidad infantil de $\gamma_{2}=0,17$ puntos. El impacto indirecto del gasto en salud en la mortalidad infantil se puede calcular como el producto del coeficiente sobre crecimiento económico de la ecuación de gasto en salud y el coeficiente sobre gasto en salud de la ecuación de crecimiento del PIB $\left(\gamma_{1} \alpha_{5}=0,76^{*} 0,47=0,35\right)$. El impacto total del gasto sanitario en la mortalidad infantil equivale a la suma de los efectos directos e indirectos $(0,52)$, lo que indica que un incremento de este gasto de 1 punto porcentual da lugar a una disminución de la tasa de mortalidad infantil de 0,52 puntos porcentuales. 
Cuadro 4

Efecto total de los gastos en salud sobre la mortalidad infantil

\begin{tabular}{lccc}
\hline & Efecto directo del HEXP en la CHM & $\begin{array}{c}\text { Efecto indirecto del HEXP en la } \\
\text { CHM vía crecimiento económico }\end{array}$ & Efecto total del HEXP en la CHM \\
\hline Coeficiente & $\gamma_{2}$ & $\gamma_{1}{ }^{*} \alpha_{5}$ & $\gamma_{1}{ }^{*} \alpha_{5}+\gamma_{2}$ \\
\hline Estimación & 0,17 & $0,76{ }^{*} 0,47=0,35$ & $0,76^{*} 0,47+0,17=0,52$ \\
\hline
\end{tabular}

Fuente: Elaboración propia.

Los resultados que se presentan en el cuadro 4 muestran claramente que el gasto sanitario tiene un impacto significativo en el estado de salud de los niños al conducir a un aumento del crecimiento económico y por consiguiente a mejores condiciones de vida en la infancia. Un aumento del gasto en salud del 1\% puede redundar en una disminución global de la tasa de mortalidad infantil de 0,52 puntos porcentuales, que se divide entre un impacto directo de 0,17 puntos porcentuales y un impacto indirecto de 0,35. Esto indica que el efecto indirecto, que se produce a través del crecimiento económico, es mayor que el efecto directo o tradicional, observado en el acceso a la atención de salud para satisfacer las necesidades médicas.

Por último, cabe mencionar que las conclusiones de este estudio inicial no parecen ser válidas para todos los países de la muestra, y los resultados podrían depender de las características estructurales de cada economía. Los hallazgos deben analizarse con cautela debido a que la muestra que se utilizó era heterogénea, pues contenía tanto países desarrollados como en desarrollo, con diferentes estructuras y estrategias económicas. De cualquier forma, la experiencia muestra que un mayor gasto de los gobiernos en salud se asocia con tasas de mortalidad infantil más bajas, mientras que un menor gasto público en este sector está relacionado con tasas más altas.

\section{B. Desagregación de la muestra y análisis de robustez}

No es apropiado llevar a cabo un estudio sobre este tema con una muestra de países que no tengan características relativamente similares, ya que no sería posible tener en cuenta la naturaleza específica de cada país y se podrían obtener resultados incorrectos que no se pueden generalizar. Una separación de la muestra podría conducir a resultados más exactos que reflejen la naturaleza heterogénea de los grupos estudiados.

En los párrafos siguientes se analizarán los resultados en términos de robustez, para lo cual, en primer lugar, se subdivide la muestra completa en cuatro más pequeñas. Esto significa que los resultados podrían ser sensibles a la elección de la muestra, de modo que el objetivo es comparar las cuatro muestras, definidas por nivel de ingreso. Conforme a la clasificación del Banco Mundial, se creó una base de datos con información del período comprendido entre 1995 y 2013, que contiene cuatro muestras con países de todo el mundo: 20 países de ingreso bajo, 23 de ingreso medio-bajo, 25 de ingreso medio-alto y 25 países de ingreso alto. A pesar de que la historia económica con seguridad será diferente de un país a otro, los países de cada grupo son similares en cuanto a sus condiciones económicas y de salud, así como en relación con las circunstancias políticas, normativas y sociales o culturales. El objetivo es señalar las consecuencias de las políticas adoptadas por cada grupo de países. Con este fin, se utilizará el mismo enfoque y la misma metodología empírica.

\section{Análisis de robustez}

En los cuadros 5, 6, 7 y 8 se presentan los resultados de los modelos de regresión correspondientes a los cuatro subgrupos de países, que se deben interpretar con prudencia. 


\section{Cuadro 5}

Países de ingresos altos:

efectos de los gastos en salud sobre la mortalidad infantil

\begin{tabular}{|c|c|c|c|}
\hline Variables & $\begin{array}{c}\text { Ecuación de } \\
\text { CHM }\end{array}$ & $\begin{array}{c}\text { Ecuación de } \\
\text { GDPG }\end{array}$ & $\begin{array}{l}\text { Ecuación de } \\
\text { HEXP }\end{array}$ \\
\hline \multirow{2}{*}{ GDPG } & $-1,214$ & - & 0,238 \\
\hline & $(-5,26)^{\star \star \star}$ & - & $(3,07)^{\star \star \star}$ \\
\hline \multirow{2}{*}{ HEXP } & $-0,32$ & 0,752 & - \\
\hline & $(-4,28)^{\star \star}$ & $(2,93)^{\star \star}$ & - \\
\hline \multirow{2}{*}{ POV } & 2,84 & - & - \\
\hline & $(2,35)^{\star \star}$ & - & - \\
\hline \multirow{2}{*}{ Water } & $-0,649$ & - & - \\
\hline & $(-2,36)^{\star \star}$ & - & - \\
\hline \multirow{2}{*}{ Life EXP } & $-2,025$ & - & - \\
\hline & $(1,72)^{*}$ & - & - \\
\hline \multirow{2}{*}{ FemL } & $-1,906$ & - & - \\
\hline & $(3,12)^{\star \star}$ & - & - \\
\hline \multirow{2}{*}{ URB } & $-0,046$ & - & - \\
\hline & $(-2,62)^{\star \star}$ & - & - \\
\hline \multirow{2}{*}{$\mathrm{CO}_{2}$} & 0,235 & - & - \\
\hline & $(2,76)^{\star *}$ & - & - \\
\hline \multirow{2}{*}{ INQ } & - & $-0,102$ & - \\
\hline & - & $(-2,67)^{\star \star}$ & - \\
\hline \multirow{2}{*}{ INF } & - & $-0,253$ & - \\
\hline & - & $(-2,89)^{\star \star}$ & - \\
\hline \multirow{2}{*}{ TRA } & - & 0,495 & - \\
\hline & - & $(2,04)^{\star \star}$ & - \\
\hline \multirow{2}{*}{ FD } & - & 0,952 & - \\
\hline & - & $(3,26)^{\star \star}$ & - \\
\hline \multirow{2}{*}{$\mathbb{R}$} & - & 2,391 & - \\
\hline & - & $(2,643)^{\star \star \star}$ & - \\
\hline \multirow{2}{*}{ LF } & - & 1,434 & - \\
\hline & - & $(2,12)^{\star \star}$ & - \\
\hline \multirow{2}{*}{ TechProg } & - & - & 3,522 \\
\hline & - & - & $(5,27)^{\star \star}$ \\
\hline \multirow{2}{*}{ DocDens } & - & - & 0,205 \\
\hline & - & - & $(2,99)^{\star \star}$ \\
\hline \multirow{2}{*}{ PopStr } & - & - & 2,523 \\
\hline & - & - & $(2,15)^{\star \star}$ \\
\hline \multirow{2}{*}{ INST } & - & - & 0,712 \\
\hline & - & - & $(3,64)^{\star \star}$ \\
\hline \multirow{2}{*}{ Constante } & 10,157 & 8,445 & 6,367 \\
\hline & $(4,33)^{\star \star \star}$ & $(4,751)^{\star \star \star}$ & $(4,37)^{\star \star}$ \\
\hline Número de observaciones & 475 & 475 & 475 \\
\hline $\mathrm{R}^{2}$ & 0,19 & 0,17 & 0,15 \\
\hline
\end{tabular}

Fuente: Elaboración propia.

Nota: $\quad$ * significativo al $10 \%$; ${ }^{\star \star}$ significativo al $5 \%$; ${ }^{\star \star \star}$ significativo al $1 \%$. 


\section{Cuadro 6}

Países de ingresos medio-altos: efectos de los gastos en salud sobre la mortalidad infantil

\begin{tabular}{|c|c|c|c|}
\hline Variables & $\begin{array}{c}\text { Ecuación de } \\
\text { CHM }\end{array}$ & $\begin{array}{l}\text { Ecuación de } \\
\text { GDPG }\end{array}$ & $\begin{array}{l}\text { Ecuación de } \\
\text { HEXP }\end{array}$ \\
\hline \multirow{2}{*}{ GDPG } & $-0,961$ & - & 0,56 \\
\hline & $(-1,99)^{\star \star}$ & - & $(2,94)^{\star \star}$ \\
\hline \multirow{2}{*}{ HEXP } & $-0,242$ & 2,16 & - \\
\hline & $(-2,19)^{\star \star}$ & $(3,45)^{\star \star}$ & - \\
\hline \multirow{2}{*}{ POV } & 0,852 & - & - \\
\hline & $(2,47)^{\star \star}$ & - & - \\
\hline \multirow{2}{*}{ Water } & $-0,239$ & - & - \\
\hline & $(-1,93)^{\star \star}$ & - & - \\
\hline \multirow{2}{*}{ Life EXP } & $-0,985$ & - & - \\
\hline & $(2,76)^{\star *}$ & - & - \\
\hline \multirow{2}{*}{ FemL } & $-1,854$ & - & - \\
\hline & $(4,18)^{\star \star}$ & - & - \\
\hline \multirow{2}{*}{ URB } & $-0,043$ & - & - \\
\hline & $(-2,41)^{\star \star}$ & - & - \\
\hline \multirow{2}{*}{$\mathrm{CO}_{2}$} & 0,213 & - & - \\
\hline & $(3,546)^{\star \star \star}$ & - & - \\
\hline \multirow{2}{*}{ INQ } & - & $-0,223$ & - \\
\hline & - & $(-1,18)$ & - \\
\hline \multirow{2}{*}{ INF } & - & $-0,262$ & - \\
\hline & - & $(-2,52)^{\star \star}$ & - \\
\hline \multirow{2}{*}{ TRA } & - & 0,341 & - \\
\hline & - & $(1,48)$ & - \\
\hline \multirow{2}{*}{ FD } & - & 1,895 & - \\
\hline & - & $(1,83)^{\star \star}$ & - \\
\hline \multirow{2}{*}{$\mathbb{R}$} & - & 2,563 & - \\
\hline & - & $(4,26)^{\star \star}$ & - \\
\hline \multirow{2}{*}{ LF } & - & 1,852 & - \\
\hline & - & $(2,31)$ & - \\
\hline \multirow{2}{*}{ TechProg } & - & - & 2,403 \\
\hline & - & - & $(2,29)^{\star \star}$ \\
\hline \multirow{2}{*}{ DocDens } & - & - & 0,133 \\
\hline & - & - & $(3,44)^{\star \star}$ \\
\hline \multirow{2}{*}{ PopStr } & - & - & 2,954 \\
\hline & - & - & $(2,05)^{\star \star \star}$ \\
\hline \multirow{2}{*}{ INST } & - & - & 0,624 \\
\hline & - & - & $(1,87)^{\star \star}$ \\
\hline \multirow{2}{*}{ Constante } & 10,524 & 13,562 & 8,52 \\
\hline & $(7,69)^{\star \star \star}$ & $(3,12)^{\star \star}$ & $(5,2)^{\star \star \star}$ \\
\hline Número de observaciones & 720 & 720 & 720 \\
\hline $\mathrm{R}^{2}$ & 0,2 & 0,19 & 0,16 \\
\hline
\end{tabular}

Fuente: Elaboración propia.

Nota: * significativo al $10 \%$; ${ }^{* \star}$ significativo al $5 \%$; ${ }^{* \star}$ significativo al $1 \%$. 


\section{Cuadro 7}

Países de ingresos medio-bajos:

efectos de los gastos en salud sobre la mortalidad infantil

\begin{tabular}{|c|c|c|c|}
\hline Variables & $\begin{array}{c}\text { Ecuación de } \\
\text { CHM }\end{array}$ & $\begin{array}{l}\text { Ecuación de } \\
\text { GDPG }\end{array}$ & $\begin{array}{c}\text { Ecuación de } \\
\text { HEXP }\end{array}$ \\
\hline \multirow{2}{*}{ GDPG } & $-0,76$ & - & 0,275 \\
\hline & $(-3,73)^{\star \star \star}$ & - & $(2,16)^{\star \star}$ \\
\hline \multirow{2}{*}{ HEXP } & $-0,318$ & 0,325 & - \\
\hline & $(-1,38)$ & $(1,76)^{*}$ & - \\
\hline \multirow{2}{*}{ POV } & 2,62 & - & - \\
\hline & $(2,91)^{\star \star}$ & - & - \\
\hline \multirow{2}{*}{ Water } & $-0,381$ & - & - \\
\hline & $(-3,74)^{\star \star}$ & - & - \\
\hline \multirow{2}{*}{ Life EXP } & $-2,82$ & - & - \\
\hline & $(-2,77)^{\star \star}$ & - & - \\
\hline \multirow{2}{*}{ FemL } & $-2,71$ & - & - \\
\hline & $(3,43)^{\star \star \star}$ & - & - \\
\hline \multirow{2}{*}{ URB } & $-0,24$ & - & - \\
\hline & $(-3,56)^{\star \star \star}$ & - & - \\
\hline \multirow{2}{*}{$\mathrm{CO}_{2}$} & 0,238 & - & - \\
\hline & $(5,645)^{\star \star \star}$ & - & - \\
\hline \multirow{2}{*}{ INQ } & - & 0,176 & - \\
\hline & - & $(1,38)$ & - \\
\hline \multirow{2}{*}{ INF } & - & $-0,254$ & - \\
\hline & - & $(-2,81)^{\star \star}$ & - \\
\hline \multirow{2}{*}{ TRA } & - & 0,351 & - \\
\hline & - & $(1,97)^{\star \star}$ & - \\
\hline \multirow{2}{*}{ FD } & - & 0,845 & - \\
\hline & - & $(1,76)^{\star *}$ & - \\
\hline \multirow{2}{*}{$\mathbb{R}$} & - & 1,543 & - \\
\hline & - & $(1,69)^{\star}$ & - \\
\hline \multirow{2}{*}{ LF } & - & 1,212 & - \\
\hline & - & $(2,11)^{\star \star}$ & - \\
\hline \multirow{2}{*}{ TechProg } & - & - & 1,263 \\
\hline & - & - & $(2,66)^{\star \star}$ \\
\hline \multirow{2}{*}{ DocDens } & - & - & 0,093 \\
\hline & - & - & $(3,17)^{\star \star}$ \\
\hline \multirow{2}{*}{ PopStr } & - & - & 2,215 \\
\hline & - & - & $(1,97)^{\star \star \star}$ \\
\hline \multirow{2}{*}{ INST } & - & - & 0,649 \\
\hline & - & - & $(1,66)^{\star}$ \\
\hline \multirow{2}{*}{ Constante } & 5,974 & 7,562 & 11,415 \\
\hline & $(5,82)^{\star \star \star}$ & $(4,95)^{\star \star}$ & $(3,51)^{\star \star \star}$ \\
\hline Número de observaciones & 552 & 552 & 552 \\
\hline $\mathrm{R}^{2}$ & 0,19 & 0,18 & 0,15 \\
\hline
\end{tabular}

Fuente: Elaboración propia.

Nota: * significativo al $10 \%$; ${ }^{* \star}$ significativo al $5 \%$; ${ }^{* \star}$ significativo al $1 \%$. 


\section{Cuadro 8}

Países de ingresos bajos:

efectos de los gastos en salud sobre la mortalidad infantil

\begin{tabular}{|c|c|c|c|}
\hline Variables & $\begin{array}{c}\text { Ecuación de } \\
\text { CHM }\end{array}$ & $\begin{array}{c}\text { Ecuación de } \\
\text { GDPG }\end{array}$ & $\begin{array}{c}\text { Ecuación de } \\
\text { HEXP }\end{array}$ \\
\hline \multirow{2}{*}{ GDPG } & $-0,082$ & - & 0,344 \\
\hline & $(-3,7)^{\star \star \star}$ & - & $(2,79)^{\star \star}$ \\
\hline \multirow{2}{*}{ HEXP } & 0,083 & 0,288 & - \\
\hline & $(1,44)$ & $(1,49)$ & - \\
\hline \multirow{2}{*}{ POV } & 0,76 & - & - \\
\hline & $(2,47)^{\star \star}$ & - & - \\
\hline \multirow{2}{*}{ Water } & $-0,204$ & - & - \\
\hline & $(-1,84)^{\star \star}$ & - & - \\
\hline \multirow{2}{*}{ Life EXP } & $-1,65$ & - & - \\
\hline & $(2,76)^{\star \star \star}$ & - & - \\
\hline \multirow{2}{*}{ FemL } & $-2,651$ & - & - \\
\hline & $(-3,18)^{\star}$ & - & - \\
\hline \multirow{2}{*}{ URB } & $-0,436$ & - & - \\
\hline & $(-2,82)^{\star \star}$ & - & - \\
\hline \multirow{2}{*}{$\mathrm{CO}_{2}$} & 0,227 & - & - \\
\hline & $(3,471)^{\star \star}$ & - & - \\
\hline \multirow{2}{*}{ INQ } & - & 0,072 & - \\
\hline & - & $(1,88)^{\star \star}$ & - \\
\hline \multirow{2}{*}{ INF } & - & $-0,262$ & - \\
\hline & - & $(-1,72)^{\star}$ & - \\
\hline \multirow{2}{*}{ TRA } & - & 0,364 & - \\
\hline & - & $(1,26)$ & - \\
\hline \multirow{2}{*}{ FD } & - & 0,865 & - \\
\hline & - & $(1,76)^{\star \star}$ & - \\
\hline \multirow{2}{*}{$\mathbb{R}$} & - & 1,485 & - \\
\hline & - & $(1,46)$ & - \\
\hline \multirow{2}{*}{ LF } & - & 1,35 & - \\
\hline & - & $(1,71)^{*}$ & - \\
\hline \multirow{2}{*}{ TechProg } & - & - & 0,26 \\
\hline & - & - & $(1,88)^{\star \star}$ \\
\hline \multirow{2}{*}{ DocDens } & - & - & 0,113 \\
\hline & - & - & $(4,49)^{\star \star}$ \\
\hline \multirow{2}{*}{ PopStr } & - & - & 2,562 \\
\hline & - & - & $(2,77)^{\star \star}$ \\
\hline \multirow{2}{*}{ INST } & - & - & 0,89 \\
\hline & - & - & $(1,26)$ \\
\hline \multirow{2}{*}{ Constante } & 4,547 & 6,541 & 3,887 \\
\hline & $(9,38)^{\star \star \star}$ & $(7,67)^{\star \star}$ & $(6,54)^{\star \star \star}$ \\
\hline Número de observaciones & 480 & 480 & 480 \\
\hline $\mathrm{R}^{2}$ & 0,19 & 0,17 & 0,15 \\
\hline
\end{tabular}

Fuente: Elaboración propia.

Nota: $\quad$ * significativo al $10 \%$; ${ }^{* \star}$ significativo al $5 \%$; ${ }^{\star \star \star}$ significativo al $1 \%$.

Los resultados indican que las variables explicativas varían según la muestra considerada. Es interesante que la regresión simple es válida para los modelos en que se utiliza la muestra de todos los países y los subgrupos de ingresos medio-altos y altos. No obstante, en el caso de los países de ingresos bajos y medio-bajos, el modelo no es capaz de explicar la relación entre la mortalidad infantil y el gasto en salud. En otras palabras, el gasto sanitario de los gobiernos tiene un efecto positivo y significativo en términos de reducir las tasas de mortalidad en los países de ingresos medio-altos y altos, pero el impacto no es estadísticamente significativo cuando se trata de países de ingresos bajos 
y medio-bajos. Este resultado es lógico ya que, de acuerdo con la literatura especializada, el gasto en salud tiene un efecto positivo sobre la mortalidad infantil cuando excede un cierto umbral que, conforme a lo que en general se supone, ha sido alcanzado en los países emergentes y desarrollados. En contraste, en los países de ingresos bajos y medio-bajos, donde los sistemas de salud todavía no son capaces de ofrecer servicios médicos a todos los segmentos de la sociedad, el gasto podría no tener efectos beneficiosos en términos de reducir la mortalidad. Esto se relaciona, además, con las deficiencias de infraestructura y personal de salud que existen en estos grupos de países, dado que los recursos que se asignan a la provisión y mantenimiento de las instalaciones son limitados. Las diferencias mencionadas también se pueden explicar por el hecho de que, en los países de ingresos medio-altos y altos, el mayor ingreso promueve la accesibilidad a mejores instalaciones de salud y a nutrición, higiene, educación y atención médica de mayor calidad. En contraste, los niños que nacen en países de ingresos bajos tienen una mayor probabilidad de experimentar problemas de salud desde el nacimiento y acumular riesgos de este tipo a medida que crecen. La educación, el cambio tecnológico, el ingreso y las diferencias culturales se señalan como factores que determinan los resultados en materia de salud, en lugar del gasto sanitario. Además, en los países menos desarrollados, donde los recursos son más escasos, generalmente el gasto en salud es menos prioritario dentro del presupuesto del gobierno.

Los individuos que pertenecen a los grupos socioeconómicos más bajos tienen también una menor probabilidad de acceder a la atención de salud: un aumento de los cobros que se hacen a los usuarios en los hospitales o clínicas afectará más a los pobres que a las personas más acomodadas. Esto es coherente con otros estudios que no revelan una relación significativa entre el gasto sanitario y la mortalidad infantil, pero que muestran que los niños nacidos en hogares de bajos ingresos probablemente experimentarán problemas de desarrollo y de salud desde el momento de nacer y tendrán más riesgos sanitarios durante su crecimiento (Filmer y Pritchett, 1999; Thornton, 2002; Roberts, 2003). Otros autores, como Viegi y Baldacci (2002), consideran que los resultados dependen del conjunto de datos y los métodos de estimación utilizados y que el impacto del gasto sanitario en la condición de salud es pequeño o estadísticamente no significativo (Filmer, Hammer y Pritchett, 1998).

El hecho de que, en los países menos desarrollados, los gastos en salud no tengan efectos significativos puede deberse a los mayores costos de la tecnología médica, mientras que otra razón de la diferencia entre los países desarrollados y en desarrollo podría ser la ineficiencia del gasto sanitario. Asimismo, la corrupción es más común en los países menos desarrollados y la prioridad que se da al gasto en salud es relativamente baja. En pequeña escala, los hogares de los países en desarrollo a menudo están atrapados en la trampa de pobreza, lo que influye en gran medida en su estado de salud deficiente. Por otra parte, las variables institucionales que reflejan la calidad de la gobernanza, como los derechos de propiedad, los procedimientos administrativos y el funcionamiento del sector público, podrían también explicar las diferencias en el acceso a los servicios médicos. Así, los niveles altos de calidad institucional son sinónimo del acceso igualitario a los servicios. Esto quiere decir que se deben incluir consideraciones relativas a la equidad en las políticas de salud pública, que en la actualidad son relativamente desfavorables para los pobres. Por lo tanto, la incapacidad del modelo para explicar la relación entre el gasto en salud y la mortalidad infantil sugiere que el gasto del gobierno no es eficiente ni eficaz. Intuitivamente, el contexto institucional parece ser al menos tan influyente como el monto del gasto sanitario y es probable que sea un factor decisivo en la relación entre gasto en salud y mortalidad infantil. Los países de ingresos medios y altos que han reducido las tasas de mortalidad han adoptado políticas basadas en el cumplimiento de los requisitos tradicionales (como límites del déficit público, infraestructura de salud y alfabetización femenina, entre otros) y, especialmente, en las condiciones para una gobernanza institucional adecuada. 


\section{Gasto público y privado en salud y mortalidad infantil}

Las conclusiones de este estudio ponen de relieve que una evaluación precisa de la influencia del gasto en salud sobre la tasa de mortalidad infantil requiere que este gasto se desglose en sus componentes público y privado. Esta sección tiene por objeto llevar a cabo esa división, junto con examinar los efectos de ambos componentes en los cuatro subgrupos de países. En el cuadro 9 se exponen los resultados, que se obtuvieron utilizando el modelo, las técnicas y el período ya mencionados. Las cifras ponen de manifiesto una marcada relación negativa entre el gasto público en salud y la tasa de mortalidad infantil en los países de ingresos bajos, medio-bajos y medio-altos, en los que un aumento de este gasto del $1 \%$ reduciría la mortalidad infantil en 0,2 puntos porcentuales en promedio. En contraste, el estudio no reveló una relación significativa entre las dos variables en los países de ingresos altos. Esto puede deberse a que, en los países menos desarrollados, el gasto público en salud se destina a proporcionar y desarrollar instalaciones y a mejorar el funcionamiento del sistema de salud.

Cuadro 9

Resumen de los efectos del gasto público y privado en salud sobre las tasas de mortalidad infantil

\begin{tabular}{lcc}
\hline & Gasto público & Gasto privado \\
\hline \multirow{2}{*}{ Países de ingresos bajos } & 0,002 & 1,26 \\
\cline { 2 - 3 } Países de ingresos medio-bajos & $(3,45)^{\star \star}$ & $(0,98)$ \\
\hline \multirow{2}{*}{ Países de ingresos medio-altos } & 0,002 & 0,03 \\
\hline \multirow{2}{*}{ Países de ingresos altos } & $(2,87)^{\star \star}$ & $(1,42)$ \\
\cline { 2 - 3 } & 0,001 & 0,002 \\
\hline
\end{tabular}

Fuente: Elaboración propia.

Nota: * significativo al $10 \%$; ${ }^{\star \star}$ significativo al $5 \%$; ${ }^{\star \star \star}$ significativo al $1 \%$.

En términos del impacto del gasto privado en salud, el estudio mostró una relación significativa de tipo negativo entre el gasto y las tasas de mortalidad infantil en los países de ingresos medio-altos y altos, pero ningún indicio de esa clase de relación en los grupos de ingresos bajos y medio-bajos. El grado en que el gasto privado afecta al sistema de salud parece depender de la eficacia de las políticas y las instituciones. Esto se asocia con los resultados expuestos por Burnside y Dollar (2004) en cuanto a que no existe una relación significativa entre el gasto privado en salud y las variaciones de la mortalidad infantil en los países menos desarrollados.

Por último, a partir de este estudio es posible concluir que, respecto del gasto en salud, el gasto público es el canal principal para mejorar la salud infantil en los países que se encuentran en las etapas iniciales del desarrollo, pero cuando un país alcanza niveles de desarrollo más altos, el gasto privado se transforma en la principal vía de influencia sobre las tasas de mortalidad infantil. Gupta, Verhoeven y Tiongson (2001) han utilizado información de 50 países para demostrar que el gasto público es más importante para la salud de los pobres en los países de ingresos bajos que en los de ingresos altos, lo que sugiere que el rendimiento del gasto sanitario es mayor en los primeros que en los últimos. 


\section{Conclusiones y consecuencias en materia de políticas}

Este estudio tiene como fin examinar el impacto del gasto en atención de la salud sobre las tasas de mortalidad infantil. Aunque la literatura acerca de este tema es extensa, los avances teóricos y empíricos conducen a conclusiones ambiguas. En ese sentido, el presente artículo pretende aclarar la interacción mencionada, para lo cual esta se examina -centrándose en el papel que cumple en ella el crecimiento económico - en 93 países desarrollados y en desarrollo, mediante un modelo de ecuaciones simultáneas y con información correspondiente al período comprendido entre 1995 y 2013. La hipótesis de la investigación fue que habría una correlación positiva entre el gasto en atención de la salud y la mortalidad infantil, de modo que un aumento del gasto redundaría en la disminución de las tasas de mortalidad de los niños menores de cinco años. Los resultados muestran que un mayor gasto sanitario tiene un efecto positivo y significativo solo en los países de ingresos medio-altos y altos, pero no en los de ingresos bajos y medio-bajos. El hecho de que este gasto no tenga un impacto significativo en estos dos últimos grupos de países podría indicar que los recursos no se están asignando en forma eficaz al sector de la salud. Las conclusiones a las que se llegó también confirman la importancia del crecimiento del PIB en cuanto a explicar tanto el gasto en salud como la mortalidad infantil. Asimismo, revelan que, en los países de menor desarrollo, el gasto público en salud tiene un efecto mayor sobre las tasas de mortalidad que el gasto privado, mientras que en los niveles de desarrollo altos el gasto privado tiene un impacto positivo en el estado de salud de los niños.

Los resultados empíricos obtenidos mediante el presente estudio, junto con el debate subsiguiente, ponen de manifiesto varias importantes lecciones y consecuencias respecto de las políticas, que son importantes para quienes participan en el diseño de programas de salud orientados a reducir las tasas de mortalidad infantil, junto con mejorar el estado de salud en general. Los gobiernos deberían aumentar la cantidad de recursos destinados a la prestación de servicios de salud. Además, la creación de alianzas público-privadas eficaces para desarrollar el sector podría significar una gran contribución a la mejora del estado de salud de la población. Para reducir las tasas de mortalidad infantil, los países deben emprender una serie de reformas. A pesar de que el gasto del gobierno puede favorecer la disminución de la mortalidad infantil en los países más ricos, el gasto en salud no debería estar confinado únicamente al gasto público, pues el gasto privado y las fuentes externas también pueden mejorar el acceso a la salud a fin de satisfacer las necesidades médicas. También se debería aumentar el gasto en educación, debido a que la alfabetización de las mujeres puede ser un factor clave respecto de la condición de salud de sus hijos y de la población en general. En resumen, la mortalidad infantil se puede disminuir mediante el fortalecimiento de los sistemas nacionales de salud, la ampliación de los programas de inmunización, el mayor control del crecimiento infantil, el aseguramiento de la supervivencia y de una mejor salud de las madres, el apoyo a una nutrición maternoinfantil más adecuada, la inversión en una mejor salud reproductiva, y el financiamiento de la infraestructura necesaria. 


\section{Bibliografía}

Baldacci, E., M. T. Guin-Siu y L. de Mello (2003), "More on the effectiveness of public spending on health care and education: a covariance structure model", Journal of International Development, vol. 15, № 6, Wiley.

Baldacci, E. y otros (2004), "Social spending, human capital, and growth in developing countries: implications for achieving the MDGs", IMF Working Paper, N 04/217, Washington, D.C., Fondo Monetario Internacional (FMI).

Barro, R. (1996), "Determinants of economic growth: a cross-country empirical study", NBER Working Paper, N 5968, Cambridge, Massachusetts, Oficina Nacional de Investigaciones Económicas.

Berger, M. C. y J. Messer (2002), "Public financing of health expenditures, insurance, and health outcomes", Applied Economics, vol. 34, N¹7, Taylor \& Francis.

Black, R. y otros (2010), "Global, regional, and national causes of child mortality in 2008: a systematic analysis", The Lancet, vol. 375, N 9730.

Bokhari, F., Y. Gai y P. Gottret (2007), "Government health expenditures and health outcomes", Health Economics, vol. 16, N³, Wiley.

Bourbonnais, R. (2002), Économétrie, París, Dunod.

Burnside, C. y D. Dollar (2004), "Aid, policies, and growth: revisiting the evidence”, Policy Research Working Paper, No WPS 3251, Washington, D.C., Banco Mundial.

Cutler, D., A. Deaton y A. Lleras-Muney (2006), "The determinants of mortality", Journal of Economic Perspectives, vol. 20, N³, Nashville, Tennessee, American Economic Association.

Dhrifi, A. (2015), "Financial development and the 'growth-inequality-poverty' triangle", Journal of the Knowledge Economy, vol. 6, $\mathrm{N}^{\circ} 4$, Springer.

(2014), "Agricultural productivity and poverty alleviation: what role for technological innovation", Journal of Economics and Social Studies, vol. 4, No 1.

Filmer, D., J. S. Hammer y L. H. Pritchett (1998), "Health policy in poor countries: weak links in the chain", Policy Research Working Paper, N 1874, Washington, D.C., Banco Mundial.

Filmer, D. y L. Pritchett (1999), "The impact of public spending on health: does money matter?", Social Science \& Medicine, vol. 49, N 10, Amsterdam, Elsevier.

(1997), "Child mortality and public spending on health: how much does money matter?", Policy Research Working Paper, N 1864, Washington, D.C., Banco Mundial.

Gupta, S. y A. Baghel (1999), "Infant mortality in the Indian slums: case studies of Calcutta metropolis and Raipur City", Population, Space and Place, vol. 5, N 5, Wiley.

Gupta, S., M. Verhoeven y E. Tiongson (2002), "The effectiveness of government spending on education and health care in developing and transition economies", European Journal of Political Economy, vol. 18, $N^{\circ} 4$, Amsterdam, Elsevier.

(2001), "Public spending on health care and the poor", IMF Working Paper, N WP/01/127, Washington, D.C., Fondo Monetario Internacional (FMI).

Harttgen, K. y M. Misselhorn (2006), "A Multilevel Approach to Explain Child Mortality and Undernutrition in South Asia and Sub-Saharan Africa" [en línea] https://www.econstor.eu/bitstream/10419/19847/1/ Misselhorn.pdf.

Khaleghian, P. y M. das Gupta (2005), "Public management and the essential public health functions", World Development, vol. 33, N 7, Amsterdam, Elsevier.

Kpodar, K. R. (2004), "Le développement financier et la problématique de réduction de la pauvreté”, Documento de Trabajo, N²00408, Clermont-Ferrand, Centre d’Études et de Recherche sur le Développement International (CERDI).

Musgrove, P. (1996), "Public and private roles in health: theory and financing patterns", HNP Discussion Paper, N 339, Washington, D.C., Banco Mundial.

Nixon, J. y P. Ulmann (2006), "The relationship between health care expenditure and health outcomes", European Journal of Health Economics, vol. 7, $\mathrm{N}^{\circ} 1$, Springer.

Nolte, E. y M. Mckee (2004), Does Health Care Save Lives? Avoidable Mortality Revisited, Londres, The Nuffield Trust.

Odhiambo, N. M. (2009), "Finance-growth-poverty nexus in South Africa: a dynamic causality linkage", The Journal of Socio-Economics, vol. 38, N², Amsterdam, Elsevier.

OMS (Organización Mundial de la Salud) (2014), Consultation on Improving Measurement of the Quality of Maternal, Newborn and Child Care in Health Facilities, Ginebra. 
(2001), "Global Public Goods for Health: New Strategies for the 21st Century", Comisión sobre Macroeconomía y Salud [en línea] http://www.cid.harvard.edu.

Pritchett, L. y L. H. Summers (1996), "Wealthier is healthier", The Journal of Human Resources, vol. 31, № 4, Madison, University of Wisconsin Press.

Ravallion, M. (1997), "Can high-inequality developing countries escape absolute poverty?", Economics Letters, vol. 56, № 1, Amsterdam, Elsevier.

Roberts, R. (2003), "Poverty reduction outcomes in education and health public expenditure and aid", Documento de Trabajo, № 210, Londres, Overseas Development Institute.

Roberts, B. W. y T. Bogg (2004), "A longitudinal study of the relationships between conscientiousness and the social-environmental factors and substance-use behaviors that influence health", Journal of Personality, vol. 72, N², Wiley.

Scheffler, R. y otros (2009), "Estimates of sub-Saharan Africa health care professional shortages in 2015", Health Affairs, vol. 28, № 5 .

Schultz, T. P. (1993), "Studying the impact of household economic and community variables on child mortality", Population and Development Review, vol. 10, Nueva York, Consejo de Población.

Thornton, J. (2002), "Estimating a health production function for the US: some new evidence", Applied Economics, vol. 34, № 1, Taylor \& Francis.

Viegi, G. y S. Baldacci (2002), "Epidemiological studies of chronic respiratory conditions in relation to urban air pollution in adults", The Impact of Air Pollution on Respiratory Health, G. d'Amato y S. T. Holgate (eds.), European Respiratory Society.

Wagstaff, A. (2002), "Poverty and health sector inequalities", Boletín de la Organización Mundial de la Salud, vol. 80, № 2, Ginebra.

Wagstaff, A., E. van Doorslaer y N. Watanabe (2003), "On decomposing the causes of health sector inequalities, with an application to malnutrition inequalities in Vietnam", Journal of Econometrics, vol. 112, $N^{\circ}$ 1, Amsterdam, Elsevier.

Wang, Y. y J. Q. Wang (2002), "A comparison of international references for the assessment of child and adolescent overweight and obesity in different populations", European Journal of Clinical Nutrition, vol. 56, NN 10, Londres, Springer Nature.

Zakir, M. y P. V. Wunnava (1997), "Factors affecting infant mortality rates: evidence from cross-sectional data", Applied Economics Letters, vol. 6, № 5, Taylor \& Francis. 